\title{
Density waves in the shearing sheet
}

\section{Swing amplification}

\begin{abstract}
B. Fuchs ${ }^{\star}$
Astronomisches Rechen-Institut, Mönchhofstraße 12-14, 69120 Heidelberg, Germany

Received 6 November 2000 / Accepted 14 December 2000

Abstract. The shearing sheet model of a galactic disk is studied anew. The theoretical description of its dynamics is based on three building blocks: stellar orbits, which are described here in epicyclic approximation, the collisionless Boltzmann equation determining the distribution function of stars in phase space, and the Poisson equation in order to take account of the self-gravity of the disk. Using these tools I develop a new formalism to describe perturbations of the shearing sheet. Applying this to the unbounded shearing sheet model I demonstrate again how the disturbances of the disk evolve always into "swing amplified" density waves, i.e. spiral-arm like, shearing density enhancements, which grow and decay while the wave crests swing by from leading to trailing orientation. Several examples are given how such "swing amplification" events are incited in the shearing sheet.
\end{abstract}

Key words. galaxies: kinematics and dynamics - galaxies: spiral

\section{Introduction}

The shearing sheet (Goldreich \& Lynden-Bell 1965; Julian \& Toomre 1966) model has been developed as a tool to study the dynamics of galactic disks and is particularly well suited to describe theoretically the dynamical mechanisms responsible for the formation of spiral arms. For the sake of simplicity the model describes only the dynamics of a patch of a galactic disk. It is assumed to be infinitesimally thin and its radial size is assumed to be much smaller than the disk. Polar coordinates can be therefore rectified to pseudo-cartesian coordinates and the velocity field of the differential rotation of the disk can be approximated by a linear shear flow. These simplifications allow an analytical treatment of the problem, which helps to clarify the underlying physical processes operating in the disk. Goldreich \& Lynden-Bell (1965) used hydrodynamical equations as dynamical equations, which were combined with the Poisson equation in order to take account of the self-gravity of the disk. Their principal result was a gravitational instability giving rise to spiral-arm like density enhancements, which take part in the general shear of the disk and grow while swinging by from leading to trailing orientation. This effect has been pursued and discussed further in many studies, notably by Goldreich \& Tremaine (1978). Independently Julian \& Toomre (1966) have investigated a shearing sheet made of stars by using the collisionless Boltzmann equation combined with the Poisson equation and found also "swing amplification" of spiral density waves with the additional effect that the density perturbations die out eventually due to smearing out of the ever tightening spiral arms by the finite epicyclic motions of the stars. Toomre (1981) has argued vigorously that this swing-amplification mechanism is the principal process of spiral arm formation in galactic disks and that the spiral arms of many spiral galaxies are thus of transient nature. Indeed, numerical simulations of the dynamical evolution of self gravitating, differentially rotating disks by Sellwood \& Carlberg (1984) have shown impressively recurring swing-amplification events, which lead to an ever-changing appearance of the disk.

The alternative concept of quasi-stationary density waves has been pursued in the very influential work of Lin, Shu, Bertin and collaborators culminating in the papers by Bertin et al. (1989a,b) and Lowe et al. (1994). In their work they always consider full disk models. One key ingredient of their recent models is the introduction of an inner reflecting Q-barrier, which shields the inner Lindblad resonance. Together with the semi-reflecting corotation zone this forms a kind of resonance cavity, in which the rigidly rotating density waves grow exponentially.

A general theory of modes of star disks has been developed by Kalnajs in a series of papers (Kalnajs 1971, 1977, 1978), which has been used by him and several other authors, notably Hunter (1992) and Pichon \& Cannon (1997) to describe spiral and bar-like perturbations of the disks. Density waves as modes of disks have been also searched for and localized in numerical simulations of the dynamical evolution of star disks by Donner \& Thomasson (1994)

\footnotetext{
* e-mail: fuchs@ari.uni-heidelberg.de
} 
and Sellwood and collaborators (see Sellwood \& Evans 2000 and references therein, also Toomre 1981). Toomre \& Kalnajs (1980) have introduced inner boundaries into their numerical, softened gravity, simulations of the evolution of the shearing sheet and describe two kind of modes growing in the sheet. The present paper is the first of a series meant as a complement to these studies. I consider again a shearing sheet made of stars and discuss first the unbounded sheet and then the dynamical consequences, when inner boundary conditions are applied. The aim is to give a consistent theoretical description of pure swing-amplification as well as exponentially growing modes in the framework of the same model. In this paper I consider the unbounded shearing sheet. The purpose is to develop the formalism, and I obtain mainly previously known results again. Contrary to previous studies I avoid Lagrangian - shearing - coordinates and stay in the Euler picture. This leads to a compact, transparent form of the central Volterra integral equation (cf. Sect. 6), and will enable me also later, when I consider a bounded shearing sheet, to derive a dispersion relation for rigidly rotating, exponentially growing modes of the disk.

\section{Stellar orbits}

The first step required in order to construct a stellardynamical model of a galactic disk is to describe the stellar orbits in the disk. The equations of motion of the stars are derived from the Lagrangian

$L=\frac{1}{2}\left(\dot{r}^{2}+r \dot{\theta}^{2}\right)-\Phi(r, \theta, z)$,

where $r, \theta$ denote polar coordinates. Since the disk is assumed to be infinitesimally thin, only plane orbits are considered. $\dot{r}, \dot{\theta}$ are the corresponding radial and angular velocities of the stars. $\Phi(r, \theta, z)$ is the gravitational potential of the disk. The potential is assumed to be axisymmetric, $\Phi(r, z)$, in the present section. Non-axisymmetric perturbations of this basic state of the disk will be taken into account in the next section. Considering now a patch of the disk as in the shearing sheet model I introduce pseudocartesian coordinates

$x=r-r_{0} \quad$ and $\quad y=r_{0}\left(\theta-\Omega_{0} t\right)$.

$r_{0}$ denotes the galactocentric distance of the center of the patch and $\Omega_{0}=\left[\frac{1}{r_{0}}\left(\frac{\mathrm{d} \Phi}{\mathrm{d} r}\right)_{r_{0}}\right]^{\frac{1}{2}}$ is the mean angular velocity of the patch around the galactic center. In order to keep the analysis tractable I consider the stellar orbits in epicyclic approximation (Lindblad 1959). This assumes that

$|x| \ll r_{0} \quad$ and $\quad|\dot{x}|,|\dot{y}| \ll \Omega_{0} r_{0}$.

Taylor expansion of the Lagrangian with respect to $x$ leads to

$L \approx \frac{1}{2}\left[r_{0}^{2} \Omega_{0}^{2}-\Phi\left(r_{0}\right)+\dot{x}^{2}+\dot{y}^{2}+4 \Omega_{0} x \dot{y}+4 A \Omega_{0} x^{2}\right]$, where $A$ denotes Oort's constant, $A=-\frac{1}{2} r_{0}\left(\frac{\mathrm{d} \Omega}{\mathrm{d} r}\right) r_{0}$. The equations of motion are then given by the standard epicyclic equations,

$\ddot{x}=2 \Omega_{0} \dot{y}+4 A \Omega_{0} x \quad$ and $\quad \ddot{y}=-2 \Omega_{0} \dot{x}$,

which can be solved straightforward in terms of linear and trigonometric functions (cf. Eqs. (10) and (11) below). The linear terms give the mean motion of the stars, whereas the trigonometric terms describe the epicyclic oscillations of the stars around the mean motion with a period of $\kappa=\sqrt{-4 \Omega_{0} B}$, where $B=A-\Omega_{0}$ denotes Oort's second constant. The plane stellar orbits conserve two integrals of motion, one related to angular momentum,

$J_{2}=\frac{\partial L}{\partial \dot{y}}=\Omega_{0} r_{0}+2 \Omega_{0} x+\dot{y}$,

and the radial action integral,

$J_{1}=\frac{1}{2 \kappa}\left(u^{2}+\frac{\kappa^{2}}{4 B^{2}} v^{2}\right)$.

The radial action integral is defined in terms of the radial velocity of a star $u=\dot{x}$ and the circumferential velocity referred to the mean circular velocity of all stars at the position of the star, $v=\dot{y}+2 A x=J_{2}-\Omega_{0} r_{0}+2 B x$. Next, the Hamiltonian of the stellar orbits is derived from the Lagrangian (4) and expressend in terms of the integrals of motion giving

$H=\kappa J_{1}+\frac{A}{2 B}\left(J_{2}-\Omega_{0} r_{0}\right)^{2}-\frac{1}{2} \Omega_{0}^{2} r_{0}^{2}$.

Angle variables $w_{1}, w_{2}$ are introduced as canonical conjugate variables to the integrals of motion,

$\dot{w}_{1}=\frac{\partial H}{\partial J_{1}}=\kappa, \dot{w}_{2}=\frac{\partial H}{\partial J_{2}}=\frac{A}{B}\left(J_{2}-\Omega_{0} r_{0}\right)$.

Assembling the results from above, the orbit equations can be written as

$x=\frac{J_{2}-\Omega_{0} r_{0}}{-2 B}+\sqrt{\frac{2 J_{1}}{\kappa}} \sin w_{1}$,

$y=w_{2}-\frac{\sqrt{2 \kappa J_{1}}}{2 B} \cos w_{1}$,

$u=\sqrt{2 \kappa J_{1}} \cos w_{1}$,

$v=\frac{2 B}{\kappa} \sqrt{2 \kappa J_{1}} \sin w_{1}$.

These equations show that the mean motions of the stars are given by

$x=\frac{J_{2}-\Omega_{0} r_{0}}{-2 B}=$ const.,

$y=\frac{A}{B}\left(J_{2}-\Omega_{0} r_{0}\right) t=-2 A x t$,

describing the linear shear flow. The trigonometric terms describe the epicyclic oscillations around the mean motion, with their amplitude determined by the radial action integral $J_{1}$. 


\section{Boltzmann equation}

The ensemble of stars is described statistically by the distribution function of stars in phase space $f$. Its time evolution is determined by the collisionless Boltzmann equation

$\frac{\partial f}{\partial t}+[f, H]=0$

where the Poisson bracket indicates the divergence of the Hamilton flow in phase space. If integrals of motion and angle variables are used the Poisson bracket has the form

$[f, H]=\frac{\partial f}{\partial w_{1}} \frac{\partial H}{\partial J_{1}}+\frac{\partial f}{\partial w_{2}} \frac{\partial H}{\partial J_{2}}$.

Following Julian \& Toomre (1966), the Schwarzschild distribution,

$f_{0}=\frac{\kappa}{-2 B} \frac{\Sigma_{0}}{2 \pi \sigma_{u}^{2}} \exp -\frac{\kappa J_{1}}{\sigma_{u}^{2}}$

is chosen as the distribution function of the homogenous background of the sheet. $\Sigma_{0}$ denotes the surface density of the disk, which is kept constant over the sheet. $\sigma_{u}$ is the radial velocity dispersion of the stars. The circumferential velocity dispersion is given according to the epicyclic ratio $\sigma_{v} / \sigma_{u}=4 B^{2} / \kappa^{2}$. Since the stationary Schwarzschild distribution depends only on the radial action integral, it solves the Boltzmann equation by definition (cf. Eq. (13)).

\section{Linear perturbation analysis}

Spiral arms are usually thought to be only minor perturbations of galactic disks. Thus I choose a perturbation "Ansatz" of the form

$f=f_{0}+\delta f, H=H_{0}+\delta \Phi$,

where $H_{0}$ is the Hamiltonian (8) of the unpertubed orbits. The physical idea is to subject the shearing sheet to a small spiral-like potential perturbation $\delta \Phi$ and to determine the disk response $\delta f$ by solving the Boltzmann equation. The Boltzmann equation is linearized accordingly,

$\frac{\partial \delta f}{\partial t}+\left[f_{0}, \delta \Phi\right]+\left[\delta f, H_{0}\right]=0$

where the quadratic Poisson bracket $[\delta f, \delta \Phi]$ has been neglected. The role of the quadratic term will be investigated in a further paper (Fuchs 2001, in preparation). A brief first report of that work was given in Fuchs (1991). The potential perturbation is Fourier transformed with respect to the spatial coordinates and I consider a single Fourier component

$\delta \Phi_{\boldsymbol{k}}=\Phi_{\boldsymbol{k}} \exp i\left(k_{x} x+k_{y} y\right)$

in Eq. (16). The Poisson brackets have then the explicit form

$\left[f_{0}, \delta \Phi_{\boldsymbol{k}}\right]=\frac{\sqrt{2 \kappa J_{1}}}{\sigma_{u}^{2}}\left[i k_{x} \cos w_{1}+\frac{\kappa}{2 B} i k_{y} \sin w_{1}\right] f_{0} \delta \Phi_{\boldsymbol{k}}$ and

$\left[\delta f, H_{0}\right]=\kappa \frac{\partial \delta f}{\partial w_{1}}+\frac{A}{B}\left(J_{2}-\Omega_{0} r_{0}\right) \frac{\partial \delta f}{\partial w_{2}}$,

which leads to the linearized Boltzmann equation

$\frac{\partial \delta f}{\partial t}+\kappa \frac{\partial \delta f}{\partial w_{1}}+\frac{A}{B}\left(J_{2}-\Omega_{0} r_{0}\right) \frac{\partial \delta f}{\partial w_{2}}$

$+\frac{\sqrt{2 \kappa J_{1}}}{\sigma_{u}^{2}}\left[i k_{x} \cos w_{1}+\frac{\kappa}{2 B} i k_{y} \sin w_{1}\right] \frac{\kappa}{-2 B}$

$\times \frac{\Sigma_{0}}{2 \pi \sigma_{u}^{2}} \exp \left(-\frac{\kappa J_{1}}{\sigma_{u}^{2}}\right) \Phi_{\boldsymbol{k}} \exp \left(i k_{x} x+i k_{y} y\right)=0$.

When $x$ and $y$ are substituted from Eqs. (10), Eq. (20) is a linear inhomogenous partial differential equation with $t$, $w_{1}$, and $w_{2}$ as independent variables. It becomes immediately clear that the coefficients of the equation do not depend on the time coordinate $t$ and the angle variable $w_{2}$. This allows a Fourier transformation of Eq. (20) with respect to $t$ and $w_{2}$, which may be written in abbreviated form as

$i \omega \delta f_{\omega, 1}+\kappa \frac{\mathrm{d} \delta f_{\omega, 1}}{\mathrm{~d} w_{1}}+\frac{A}{B}\left(J_{2}-\Omega_{0} r_{0}\right) i l \delta f_{\omega, 1}$

$=\mathrm{e}^{i l w_{2}} \int_{-\infty}^{\infty} \mathrm{d} w_{2}^{\prime} \mathrm{e}^{-i l w_{2}^{\prime}} \operatorname{Inhom}_{\omega}\left(J_{1}, J_{2}, w_{1}\right) \mathrm{e}^{i k_{y} w_{2}^{\prime}}$

$=2 \pi \mathrm{e}^{i l w_{2}} \operatorname{Inhom}_{\omega}\left(J_{1}, J_{2}, w_{1}\right) \delta\left(k_{y}-l\right)$,

where $\delta\left(k_{y}-l\right)$ denotes a delta function. Equation (20) will be solved explicitly below. However, it becomes clear from Eq. (21) that the solution $f_{\omega, l}$ must be proportional to $\Phi_{\boldsymbol{k}} \mathrm{e}^{i l w_{2}} \delta\left(k_{\mathrm{y}}-l\right)$, because of the linearity of the equation and the consideration that only a non-zero perturbation leads to a disk response. Transforming back to $w_{2}$ space, this implies a functional dependence of $\delta f$ on $w_{2}$ as

$\delta f \propto \mathrm{e}^{i k_{y} w_{2}}$.

Furthermore it is advantageous to split off the background distribution function from the disk response, $\delta f_{\omega}=f_{1, \omega} f_{0}$, and I obtain the ordinary differential equation

$i \omega f_{1, \omega}+\kappa \frac{\mathrm{d} f_{1, \omega}}{\mathrm{d} w_{1}}+i k_{y} \frac{A}{B}\left(J_{2}-\Omega_{0} r_{0}\right) f_{1, \omega}$

$+\frac{\sqrt{2 \kappa J_{1}}}{\sigma_{u}^{2}}\left[i k_{x} \cos w_{1}+\frac{\kappa}{2 B} i k_{y} \sin w_{1}\right] \Phi_{\boldsymbol{k}, \omega}$

$\times \exp \left\{i k_{x}\left[\frac{J_{2}-\Omega_{0} r_{0}}{-2 B}+\sqrt{\frac{2 J_{1}}{\kappa}} \sin w_{1}\right]\right.$

$\left.-i k_{y} \frac{\sqrt{2 \kappa J_{1}}}{2 B} \cos w_{1}\right\}=0$.

Equation (23) can be solved by "variation of the constant". Very similar equations have been treated with the same method by Lin et al. (1969) and Julian \& Toomre (1966) and I refer in particular to the lecture notes by Lin (1970) for a full explanation of the technique. The homogeneous part of Eq. (23) has solutions

$f_{1, \omega} \propto \exp -\frac{i}{\kappa}\left(\omega+\frac{A}{B} k_{y}\left(J_{2}-\Omega_{0} r_{0}\right)\right) w_{1}$, 
and "variation of the constant" means to look for a particular solution of Eq. (23) in the form

$f_{1, \omega}=C\left(w_{1}\right) \exp -\frac{i}{\kappa}\left(\omega+\frac{A}{B} k_{y}\left(J_{2}-\Omega_{0} r_{0}\right)\right) w_{1}$.

Inserting this into Eq. (23) and solving for $C\left(w_{1}\right)$ gives

$$
\begin{aligned}
C\left(w_{1}\right)= & -\frac{i \xi}{\sigma_{u}^{2}} \exp \left(i k_{x} \frac{J_{2}-\Omega_{0} r_{0}}{-2 B}\right) \Phi_{\boldsymbol{k}, \omega} \exp i \eta \bar{w} \\
& \times \int_{0}^{w_{1}-\bar{w}} \mathrm{~d} w_{1}^{\prime} \cos w_{1}^{\prime} \exp i\left[\xi \sin w_{1}+\eta w_{1}^{\prime}\right]
\end{aligned}
$$

where the abbreviations $\xi=\sqrt{\frac{2 J_{1}}{\kappa}} \sqrt{k_{x}^{2}+\frac{\kappa^{2}}{4 B^{2}} k_{y}^{2}}$ and $\eta=$ $\frac{1}{\kappa}\left(\omega+k_{y} \frac{A}{B}\left(J_{2}-\Omega_{0} r_{0}\right)\right)$ have been introduced to keep the formulae compact. $\bar{w}$ is defined as $\bar{w}=\arctan \left(\frac{\kappa}{2 B} \frac{k_{y}}{k_{x}}\right)$. The general solution of Eq. (23) is then given by the particular integral (25) plus the solution of the homogeneous equation multiplied by an arbitrary integration constant,

$$
\begin{aligned}
& f_{1, \omega}=\frac{\Phi_{\boldsymbol{k}, \omega}}{\sigma_{u}^{2}} \exp \left(i k_{x} \frac{J_{2}-\Omega_{0} r_{0}}{-2 B}\right)\{D-i \xi \exp i \eta \bar{w} \\
& \left.\times \int_{0}^{w_{1}-\bar{w}} \mathrm{~d} w_{1}^{\prime} \cos w_{1}^{\prime} \exp i\left[\xi \sin w_{1}^{\prime}+\eta w_{1}^{\prime}\right]\right\} \exp -i \eta w_{1} .
\end{aligned}
$$

The integration constant $D$ is determined by the requirement that the distribution function must be defined in a unique way in velocity space, i.e. must be periodic with respect to the angle variable $w_{1}$ with a period of $2 \pi$,

$f_{1, \omega}\left(w_{1}\right)=f_{1, \omega}\left(w_{1}+2 \pi\right)$.

Some algebra as indicated in Lin (1970) leads to the final result

$$
\begin{aligned}
& f_{1, \omega}= \\
& -\frac{1}{\sigma_{u}^{2}} \exp \left(i k_{x} \frac{J_{2}-\Omega_{0} r_{0}}{-2 B}\right) \Phi_{\boldsymbol{k}, \omega} \exp \left(i \xi \sin \left(w_{1}-\bar{w}\right)\right) \\
& \times\left\{1-\frac{\eta}{2 \sin (\pi \eta)} \int_{-\pi}^{+\pi} \mathrm{d} w_{1}^{\prime} \exp i\left[\eta w_{1}^{\prime}-\xi \sin w_{1}^{\prime}\right.\right. \\
& \left.\left.\times \cos \left(w_{1}-\bar{w}\right)-\xi\left(1+\cos w_{1}^{\prime}\right) \sin \left(w_{1}-\bar{w}\right)\right]\right\} .
\end{aligned}
$$

The disk response to the single Fourier component of the potential perturbation is thus given by

$\delta f=f_{0} f_{1, \omega} \exp \left(i \omega t+i k_{y} w_{2}\right)$.

Note that according to Eqs. (10) and the definitions of $\xi$ and $\bar{w}$

$k_{x} \frac{J_{2}-\Omega_{0} r_{0}}{-2 B}+\xi \sin \left(w_{1}-\bar{w}\right)+k_{y} w_{2}=k_{x} x+k_{y} y$.

This shows that the disk response follows to a first approximation the spatial pattern of the potential perturbation. However, the second term in the curly brackets in Eq. (29) depends through $\eta$ on $\left(J_{2}-\Omega_{0} r_{0}\right)$ and thus on the spatial coordinate $x$, so that contrary to $\Phi_{k, \omega}, f_{1, \omega}$ is not a Fourier component of a Fourier decomposition of the disk response.

\section{Self consistent perturbations}

The shearing sheet is assumed to be self-gravitating. Thus the potential perturbation and the corresponding disk response have to satisfy the Poisson equation,

$\left\{\frac{\partial}{\partial x^{2}}+\frac{\partial}{\partial y^{2}}+\frac{\partial}{\partial z^{2}}\right\} \delta \Phi=4 \pi G \delta \Sigma \delta(z)$,

where $\delta \Sigma$ denotes the surface density of the disk response and $\delta(z)$ is a delta function with respect to the vertical spatial coordinate $z$. $G$ is the constant of gravity. Background potentials do not enter into Eq. (32) because of the linearity of the Poisson equation. Off the midplane the right hand side of Eq. (32) is equal to zero. This leads immediately to a three-dimensional extension of a potential perturbation (17) in the form

$\delta \Phi_{\boldsymbol{k}}=\Phi_{\boldsymbol{k}} \exp \left[i\left(k_{x} x+k_{y} y\right)-\sqrt{k_{x}^{2}+k_{y}^{2}}|z|\right]$.

Integrating both sides of the Poisson equation with respect to $z$ gives the surface density required to sustain the potential (33),

$$
\begin{aligned}
& \int_{-\infty}^{+\infty} \mathrm{d} z\left\{\frac{\partial^{2}}{\partial x^{2}}+\frac{\partial^{2}}{\partial y^{2}}+\frac{\partial^{2}}{\partial z^{2}}\right\} \Phi_{\boldsymbol{k}} \\
& \times \exp \left[i\left(k_{x} x+k_{y} y\right)-\sqrt{k_{x}^{2}+k_{y}^{2}}|z|\right] \\
& =-2 \sqrt{k_{x}^{2}+k_{y}^{2}} \Phi_{\boldsymbol{k}} \exp i\left(k_{x} x+k_{y} y\right) \\
& =4 \pi G \Sigma_{\boldsymbol{k}} \exp i\left(k_{x} x+k_{y} y\right)
\end{aligned}
$$

where $\Sigma_{\boldsymbol{k}}$ denotes the Fourier coefficients of the Fourier transform of $\delta \Sigma$.

It was pointed out in the previous section that the disk response to a single Fourier component of a general potential perturbation as considered in Eqs. (17) and (33) is not a Fourier component of the disk response to the general potential perturbation. Kalnajs (1971) has shown a way to overcome this difficulty by taking Fourier transforms of both sides of the Poisson equation in the following way

$$
\begin{aligned}
& \frac{1}{(2 \pi)^{2}} \int_{-\infty}^{+\infty} \mathrm{d} z \int_{-\infty}^{+\infty} \mathrm{d} x \int_{-\infty}^{+\infty} \mathrm{d} y \mathrm{e}^{-i\left(k_{x}^{\prime} x+k_{y}^{\prime} y\right)} \\
& \left\{\frac{\partial^{2}}{\partial x^{2}}+\frac{\partial^{2}}{\partial y^{2}}+\frac{\partial^{2}}{\partial z^{2}}\right\} \int_{-\infty}^{+\infty} \mathrm{d} k_{x} \int_{-\infty}^{+\infty} \mathrm{d} k_{y} \Phi_{k} \\
& \times \exp \left[i\left(k_{x} x+k_{y} y\right)-\sqrt{k_{x}^{2}+k_{y}^{2}}|z|\right] \\
& =\frac{4 \pi G}{(2 \pi)^{2}} \int_{-\infty}^{+\infty} \mathrm{d} x \int_{-\infty}^{+\infty} \mathrm{d} y \mathrm{e}^{-i\left(k_{x}^{\prime} x+k_{y}^{\prime} y\right)} \\
& \int_{-\infty}^{+\infty} \mathrm{d} k_{x} \int_{-\infty}^{+\infty} \mathrm{d} k_{y} \int_{-\infty}^{+\infty} \mathrm{d} u \int_{-\infty}^{+\infty} \mathrm{d} v f_{0} f_{1, \omega} \mathrm{e}^{i k_{y} w_{2}} .
\end{aligned}
$$

The $k_{x}, k_{y}$ integrations indicate that all Fourier coefficients have to be considered together, while the integrations of the distribution function of the disk response over the $u$ und $v$ velocity components on the right hand side of Eq. (35) give the surface density of the disk response. 
The left hand side of Eq. (35) can be evaluated by carrying out first the quadrature with respect to $z$, giving

$$
\begin{array}{r}
-\frac{2}{(2 \pi)^{2}} \int_{-\infty}^{+\infty} \mathrm{d} x \int_{-\infty}^{+\infty} \mathrm{d} y \int_{-\infty}^{+\infty} \mathrm{d} k_{x} \int_{-\infty}^{+\infty} \mathrm{d} k_{y} \\
\times \sqrt{k_{x}^{2}+k_{y}^{2}} \Phi_{\boldsymbol{k}} \exp i\left[\left(k_{x}-k_{x}^{\prime}\right) x+\left(k_{y}-k_{y}^{\prime}\right) y\right] \\
=-2 \sqrt{k_{x}^{\prime 2}+k_{y}^{\prime 2}} \Phi_{\boldsymbol{k}^{\prime}} .
\end{array}
$$

Equation (35) represents in effect an integral equation which has to be solved to obtain self consistent pairs of potential and density perturbations of the sheet.

\section{Derivation of the Volterra integral equation}

In order to derive the central integral equation of this study the right hand side of Eq. (35) has to be evaluated explicitly. The multiple integrals imply an integration over the entire phase space and it is convenient to change the integrations from spatial $x, y$ and $u, v$ velocity coordinates to $J_{1}, J_{2}$ and $w_{1}, w_{2}$ variables. Using Eqs. (10) it can be shown that the Jacobian of the transformation is equal to one,

$\left|\frac{\partial(x, y, u, v)}{\partial\left(J_{1}, J_{2}, w_{1}, w_{2}\right)}\right|=1$.

Inserting $f_{0}, f_{1, \omega}, x$, and $y$ into the right hand side of Eq. (35) leads to

$-\frac{4 \pi G}{(2 \pi)^{2}} \int_{0}^{2 \pi} \mathrm{d} w_{1} \int_{-\infty}^{+\infty} \mathrm{d} w_{2} \int_{0}^{\infty} \mathrm{d} J_{1} \int_{-\infty}^{+\infty} \mathrm{d} J_{2} \frac{\kappa}{-2 B}$

$\times \frac{\Sigma_{0}}{2 \pi \sigma_{u}^{2}} \exp -\left(\frac{\kappa}{\sigma_{u}^{2}} J_{1}\right) \int_{-\infty}^{+\infty} \mathrm{d} k_{x} \int_{-\infty}^{+\infty} \mathrm{d} k_{y} \frac{\Phi_{\boldsymbol{k}}}{\sigma_{u}^{2}}$

$\times \exp i\left[k_{x} \frac{J_{2}-\Omega_{0} r_{0}}{-2 B}+k_{y} w_{2}+\xi \sin \left(w_{1}-\bar{w}\right)\right]$

$\times\left\{1-\frac{\eta}{2 \sin (\pi \eta)} \int_{-\infty}^{+\infty} \mathrm{d} w_{1}^{\prime} \exp i\left[\eta w_{1}^{\prime}-\xi \sin w_{1}^{\prime}\right.\right.$

$\left.\left.\times \cos \left(w_{1}-\bar{w}\right)-\xi\left(1+\cos w_{1}^{\prime}\right) \sin \left(w_{1}-\bar{w}\right)\right]\right\}$

$\times \exp -i\left[k_{x}^{\prime} \frac{J_{2}-\Omega_{0} r_{0}}{-2 B}+k_{x}^{\prime} \sqrt{\frac{2 J_{1}}{\kappa}} \sin w_{1}\right.$

$\left.+k_{y}^{\prime} w_{2}-k_{y}^{\prime} \frac{\sqrt{2 \kappa J_{1}}}{2 B} \cos w_{1}\right]$.

All of the quadratures but one are, even if tedious, straightforward. The integration over the angle variable $w_{2}$ is particularly simple,

$\frac{1}{2 \pi} \int_{-\infty}^{+\infty} \mathrm{d} w_{2} \mathrm{e}^{i\left(k_{y}-k_{y}^{\prime}\right) w_{2}}=\delta\left(k_{y}-k_{y}^{\prime}\right)$

and the integration over $k_{y}$ contracts the integrand of (38) to $k_{y}=k_{y}^{\prime}$. This shows that the integral Eq. (35) is separating in the circumferential wave numbers $k_{y}$.
The integrand of Eq. (38) is split according to the two terms in the curly bracket into two parts. The $w_{1}$ integration of the first part of the integrand leads to a quadrature

$$
\begin{array}{r}
\int_{0}^{2 \pi} \mathrm{d} w_{1} \exp i\left[\xi \sin \left(w_{1}-\bar{w}\right)-\xi^{\prime} \sin \left(w_{1}-\bar{w}^{\prime}\right)\right] \\
=\int_{0}^{2 \pi} \mathrm{d} w_{1} \exp i\left[\left(\xi^{2}-2 \xi \xi^{\prime} \cos \left(\bar{w}-\bar{w}^{\prime}\right)+{\xi^{\prime}}^{2}\right)^{\frac{1}{2}}\right. \\
\left.\times \sin \left(w_{1}-\overline{\bar{w}}\right)\right],
\end{array}
$$

where $\xi^{\prime}$ and $\bar{w}^{\prime}$ are defined analogous to $\xi$ and $\bar{w}$ for primed wave numbers. $\overline{\bar{w}}$ is given by $\overline{\bar{w}}=\arctan \left(\left(\xi \sin \bar{w}-\xi^{\prime} \sin \bar{w}^{\prime}\right) /\left(\xi \cos \bar{w}-\xi^{\prime} \cos \bar{w}^{\prime}\right)\right)$.

A Fourier expansion of the integrand of (40) with respect to $w_{1}$ (Abramowitz \& Stegun 1968, Sect. 9.1) gives immediately the result of the quadrature

$2 \pi J_{0}\left(\left(\xi^{2}-2 \xi \xi^{\prime} \cos \left(\bar{w}-\bar{w}^{\prime}\right)+\xi^{\prime 2}\right)^{\frac{1}{2}}\right)$,

where $J_{0}$ denotes the Bessel function of the first kind. The second part of the integrand of (38) is slightly more complicated,

$$
\begin{aligned}
& \int_{0}^{2 \pi} \mathrm{d} w_{1} \exp i\left[-\xi \sin \left(w_{1}^{\prime}+w_{1}-\bar{w}\right)-\xi^{\prime} \sin \left(w_{1}-\bar{w}^{\prime}\right)\right] \\
& =2 \pi J_{0}\left(\left(\xi^{2}+2 \xi \xi^{\prime} \cos \left(w_{1}^{\prime}-\bar{w}+\bar{w}^{\prime}\right)+\xi^{\prime 2}\right)^{\frac{1}{2}}\right)
\end{aligned}
$$

I consider next the integration over the action variable $J_{1}$ which leads for the first part of the integrand of (38) to a quadrature

$$
\begin{aligned}
\int_{0}^{\infty} \mathrm{d} J_{1} & \exp -\left(\frac{\kappa}{\sigma_{u}^{2}} J_{1}\right) 2 \pi J_{0}\left(\left[\frac{2}{\kappa}\left(k_{x}^{2}+\frac{\kappa^{2}}{4 B^{2}} k_{y}^{2}\right)\right.\right. \\
+ & \frac{2}{\kappa}\left(k_{x}^{\prime 2}+\frac{\kappa^{2}}{4 B^{2}} k_{y}^{\prime 2}\right)-\frac{4}{\kappa} \sqrt{k_{x}^{2}+\frac{\kappa^{2}}{4 B^{2}} k_{y}^{2}} \\
& \left.\left.\times \sqrt{k_{x}^{\prime 2}+\frac{\kappa^{2}}{4 B^{2}} k_{y}^{\prime 2}} \cos \left(\bar{w}-\bar{w}^{\prime}\right)\right]^{\frac{1}{2}} \sqrt{J_{1}}\right),
\end{aligned}
$$

if $\xi$ and $\xi^{\prime}$ are inserted into the argument of the Bessel function. According to formula (6.631) of Gradshteyn \& Ryzhik (1965) this can be evaluated as

$$
\begin{aligned}
& \frac{2 \pi \sigma_{u}^{2}}{\kappa} \exp -\frac{\sigma_{u}^{2}}{\kappa^{2}}\left[\frac{1}{2}\left(k_{x}^{2}+\frac{\kappa^{2}}{4 B^{2}} k_{y}^{2}\right)+\frac{1}{2}\left(k_{x}^{\prime 2}+\frac{\kappa^{2}}{4 B^{2}} k_{y}^{\prime 2}\right)\right. \\
& \left.-\sqrt{k_{x}^{2}+\frac{\kappa^{2}}{4 B^{2}} k_{y}^{2}} \sqrt{k_{x}^{\prime 2}+\frac{\kappa^{2}}{4 B^{2}} k_{y}^{\prime 2}} \cos \left(\bar{w}-\bar{w}^{\prime}\right)\right]
\end{aligned}
$$

and similarly for the second part of the integrand of (38)

$$
\begin{aligned}
& \frac{2 \pi \sigma_{u}^{2}}{\kappa} \exp -\frac{\sigma_{u}^{2}}{\kappa^{2}}\left[\frac{1}{2}\left(k_{x}^{2}+\frac{\kappa^{2}}{4 B^{2}} k_{y}^{2}\right)+\frac{1}{2}\left(k_{x}^{\prime 2}+\frac{\kappa^{2}}{4 B^{2}} k_{y}^{\prime 2}\right)\right. \\
& -\sqrt{k_{x}^{2}+\frac{\kappa^{2}}{4 B^{2}} k_{y}^{2}} \sqrt{k_{x}^{\prime 2}+\frac{\kappa^{2}}{4 B^{2}} k_{y}^{\prime 2}} \\
& \left.\times \cos \left(w_{1}^{\prime}-\bar{w}+\bar{w}^{\prime}\right)\right] .
\end{aligned}
$$


In the second part of the integrand of (38) a quadrature over the auxiliary angle variable $w_{1}^{\prime}$ has to be carried out,

$\int_{-\pi}^{+\pi} \mathrm{d} w_{1}^{\prime} \exp i\left[\eta w_{1}^{\prime}-\Lambda \cos \left(w_{1}^{\prime}-\bar{w}+\bar{w}^{\prime}\right)\right]$,

where I have introduced $\Lambda$ as an abbreviation for $\Lambda=$ $\frac{\sigma_{u}^{2}}{\kappa^{2}} \sqrt{k_{x}^{2}+\frac{\kappa^{2}}{4 B^{2}} k_{y}^{2}} \sqrt{k_{x}^{\prime 2}+\frac{\kappa^{2}}{4 B^{2}} k_{y}^{\prime 2}}$. A Fourier transform with respect to $w_{1}^{\prime}$ gives (Abramowitz \& Stegun 1968; Sect. 9.6)

$$
\begin{aligned}
& \int_{-\pi}^{+\pi} \mathrm{d} w_{1}^{\prime}\left(\cos \left(\eta w_{1}^{\prime}\right)+i \sin \left(\eta w_{1}^{\prime}\right)\right) \\
& \times\left[I_{0}(-\Lambda)+2 \sum_{n=1}^{\infty} I_{n}(-\Lambda) \cos \left(n\left(w_{1}^{\prime}-\bar{w}+\bar{w}^{\prime}\right)\right)\right] \\
& =I_{0}(\Lambda) \frac{2 \sin (\pi \eta)}{\eta} \\
& +2 \sum_{n=1}^{\infty} I_{n}(-\Lambda)(-1)^{n} \sin (\pi \eta) \cos \left(n\left(\bar{w}-\bar{w}^{\prime}\right)\right) \\
& \times\left[\frac{1}{\eta-n}+\frac{1}{\eta+n}\right] \\
& +2 i \sum_{n=1}^{\infty} I_{n}(-\Lambda)(-1)^{n} \sin (\pi \eta) \sin \left(n\left(\bar{w}-\bar{w}^{\prime}\right)\right) \\
& \times\left[\frac{1}{\eta-n}-\frac{1}{\eta+n}\right],
\end{aligned}
$$

where the $I_{n}$ denote modified Bessel functions of the first kind.

This leaves finally the integration over the variable $J_{2}$. The first part of the integrand of (38) is evaluated easily,

$$
\begin{aligned}
& \int_{-\infty}^{+\infty} \mathrm{d} J_{2} \exp \left[i\left(k_{x}-k_{x}^{\prime}\right) \frac{J_{2}-\Omega_{0} r_{0}}{-2 B}\right] \\
& =2 \pi(-2 B) \exp \left[i\left(k_{x}-k_{x}^{\prime}\right) \frac{\Omega_{0} r_{0}}{2 B}\right] \delta\left(k_{x}-k_{x}^{\prime}\right) .
\end{aligned}
$$

The integration over $k_{x}$ contracts the first part of the integrand of (38) to $k_{x}=k_{x}^{\prime}$.

However the second part of the integrand needs more care. The first term of the series (47) leads to

$$
\begin{aligned}
& \int_{-\infty}^{+\infty} \mathrm{d} J_{2} \exp \left[i\left(k_{x}-k_{x}^{\prime}\right) \frac{J_{2}-\Omega_{0} r_{0}}{-2 B}\right](-1) I_{0}(\Lambda) \\
& =-2 \pi(-2 B) \exp \left[i\left(k_{x}-k_{x}^{\prime}\right) \frac{\Omega_{0} r_{0}}{2 B}\right] I_{0}(\Lambda) \delta\left(k_{x}-k_{x}^{\prime}\right)
\end{aligned}
$$

and the integration over $k_{x}$ contracts this part of the second half of the integrand again to $k_{x}=k_{x}^{\prime}$. The next terms of the series (47) lead to integrals of the form

$$
\begin{aligned}
& \int_{-\infty}^{+\infty} \mathrm{d} J_{2} \exp \left[i\left(k_{x}-k_{x}^{\prime}\right) \frac{J_{2}-\Omega_{0} r_{0}}{-2 B}\right] \\
& \times(-\eta)\left[\frac{1}{\eta-n} \pm \frac{1}{\eta+n}\right] .
\end{aligned}
$$

The auxiliary variable $\eta$ is substituted for the variable $J_{2}$ and we have $\mathrm{d} \eta=\frac{A}{B} \frac{k_{y}^{\prime}}{\kappa} \mathrm{d} J_{2}$. Since the Oort constant $B$ is negative, I consider first the case of negative wave numbers $k_{y}^{\prime}$. The integral (50) is of the type

$\lim _{\lambda \rightarrow 0} \int_{-\infty+\frac{i \lambda}{\kappa}}^{+\infty+\frac{i \lambda}{\kappa}} \mathrm{d} \eta \mathrm{e}^{i \Delta \eta} \frac{\eta}{\eta \pm n}$,

where $\Delta$ denotes $\Delta=\kappa\left(k_{x}^{\prime}-k_{x}\right) /\left(2 A k_{y}^{\prime}\right)$. According to its definition the variable $\eta$ is related to the frequency $\omega$. I have assumed in the Fourier transforms throughout the previous sections a real frequency $\omega$. However, according to Landau's rule this must be understood as a limiting value of frequencies with negative imaginary parts $\lambda$. The reasoning is that only perturbations, which grow in time from minus infinity are physically relevant, and, if perturbations with other imaginary parts are considered, these must be analytical continuations of the former (cf. Binney \& Tremaine 1987). According to the definition of the Fourier transform used here the functional dependence of the perturbations is $\mathrm{e}^{i \omega t} \propto \mathrm{e}^{-\lambda t}$, so that negative imaginary parts $\lambda$ indicate growing perturbations. This implies that in the integral (51) the integration contour must be chosen in that way that it encompasses the poles of the integrand in the complex domain of $\eta$ below the real axis. With these caveats in mind the integral (51) can be evaluated as

$$
\begin{aligned}
& \lim _{\lambda \rightarrow 0} \int_{-\infty+\frac{i \lambda}{\kappa}}^{+\infty+\frac{i \lambda}{\kappa}} \mathrm{d} \eta^{\prime} \mathrm{e}^{i \Delta\left(\eta^{\prime} \mp n\right)} \frac{\eta^{\prime} \mp n}{\eta^{\prime}} \\
& =2 \pi \mathrm{e}^{\mp i \Delta n} \delta(\Delta) \mp n \mathrm{e}^{\mp i \Delta n} \lim _{\lambda \rightarrow 0} \int_{-\infty+\frac{i \lambda}{\kappa}}^{+\infty+\frac{i \lambda}{\kappa}} \mathrm{d} \eta^{\prime} \frac{\mathrm{e}^{i \Delta \eta^{\prime}}}{\eta^{\prime}} \\
& =2 \pi \mathrm{e}^{\mp i \Delta n} \delta(\Delta) \mp n \mathrm{e}^{\mp i \Delta n} \\
& \times\left(i \pi+i \mathcal{P} \int_{-\infty}^{+\infty} \mathrm{d} \eta^{\prime} \frac{\sin \left(\Delta \eta^{\prime}\right)}{\eta^{\prime}}\right)
\end{aligned}
$$

where $\eta^{\prime}$ has been substituted for $\eta^{\prime}=\eta \pm n$. $\mathcal{P}$ indicates the Cauchy principal value of the integral, and if the latter is evaluated as $\pi i \operatorname{sign}(\Delta)$ (Gradshteyn \& Ryzhik 1965, Eq. (3.722)), the integral (51) is equal to

$2 \pi \mathrm{e}^{\mp i \Delta n}(\delta(\Delta) \mp i n u(\Delta))$,

where $u(\Delta)$ denotes the unit step function,

$u(\Delta)=\left\{\begin{array}{l}1, \Delta>0 \\ \frac{1}{2}, \Delta=0 \\ 0, \Delta<0\end{array}\right.$.

Inserting this back into Eq. (50) gives

$$
\begin{aligned}
& -2 \pi \frac{B}{A} \frac{\kappa}{k_{y}^{\prime}} \exp -\left(\frac{i \omega}{\kappa} \Delta\right) \\
& \times\left[\begin{array}{cc}
\delta(\Delta) \cos (n \Delta) & -n \sin (n \Delta) u(\Delta) \\
0 & +i n \cos (n \Delta) u(\Delta)
\end{array}\right],
\end{aligned}
$$

where the upper row refers to the plus sign and the lower to the minus sign in Eq. (50), respectively. The case of positive wave numbers $k_{y}^{\prime}$ can be treated in an analogous 
way, leading to a sign reversal,

$$
\begin{aligned}
& +2 \pi \frac{B}{A} \frac{\kappa}{k_{y}^{\prime}} \exp -\left(\frac{i \omega}{\kappa} \Delta\right) \\
& \times\left[\begin{array}{cc}
\delta(\Delta) \cos (n \Delta) & -n \sin (n \Delta) u(\Delta) \\
0 & +i n \cos (n \Delta) u(\Delta)
\end{array}\right] .
\end{aligned}
$$

In order to assemble the results I consider first terms proportional $\delta\left(k_{x}-k_{x}^{\prime}\right)$ or $\delta(\Delta)$ and integrate immediately over $k_{x}$, which contracts the integrand to $k_{x}=k_{x}^{\prime}$. Equations (48), (49), and (55) together with (47) give

$$
\begin{aligned}
& -\frac{4 \pi G}{2 \pi} \frac{\kappa}{-2 B} \frac{\Sigma_{0}}{\kappa \sigma_{u}^{2}} \Phi_{k^{\prime}}\left[2 \pi(-2 B)-2 \pi(-2 B) \mathrm{e}^{-\Lambda\left(k_{x}=k_{x}^{\prime}\right)}\right. \\
& \left.\times\left\{I_{0}\left(\Lambda\left(k_{x}=k_{x}^{\prime}\right)\right)-2 \sum_{n=1}^{\infty}(-1)^{n} I_{n}\left(-\Lambda\left(k_{x}=k_{x}^{\prime}\right)\right)\right\}\right] .
\end{aligned}
$$

Since

$$
\sum_{n=1}^{\infty}(-1)^{n} I_{n}(-\Lambda)=\sum_{n=1}^{\infty} I_{\mathrm{n}}(\Lambda)=\frac{1}{2}\left(\mathrm{e}^{\Lambda}-I_{0}(\Lambda)\right)
$$

(Abramowitz \& Stegun 1968, Sect. 9.6), the term (57) cancels out exactly. This leaves according to Eqs. (47) and (56)

$$
\begin{aligned}
& -\frac{4 \pi G}{2 \pi} \frac{\kappa}{-2 B} \frac{\Sigma_{0}}{\kappa \sigma_{u}^{2}} \int_{-\infty}^{+\infty} \mathrm{d} k_{x} \Phi_{\boldsymbol{k}} \\
& \times \exp -\frac{\sigma_{u}^{2}}{\kappa^{2}}\left[\frac{1}{2}\left(k_{x}^{2}+k_{x}^{\prime 2}\right)+\frac{\kappa^{2}}{4 B^{2}} k_{y}^{\prime 2}\right] 2 \pi \frac{B}{A} \frac{\kappa}{\left|k_{y}^{\prime}\right|} \\
& \times \exp -\left(\frac{i \omega}{\kappa} \Delta\right) \\
& \times 2\left[\sum_{n=1}^{\infty}(-n) \sin (n \Delta) \cos \left(n\left(\bar{w}-\bar{w}^{\prime}\right)\right)(-1)^{n} I_{n}(-\Lambda)\right. \\
& \left.-n \cos (n \Delta) \sin \left(n\left(\bar{w}-\overline{w^{\prime}}\right)\right)(-1)^{n} I_{n}(-\Lambda)\right] u(\Delta) .
\end{aligned}
$$

The series

$\sum_{n=1}^{\infty} n \sin \left(n\left(\Delta+\bar{w}-\bar{w}^{\prime}\right)\right) I_{n}(\Lambda)$

can be simplified by using the identity

$n I_{n}(\Lambda)=\frac{\Lambda}{2}\left(I_{n-1}(\Lambda)-I_{n+1}(\Lambda)\right)$

and rearranging it as

$$
\begin{aligned}
& \frac{\Lambda}{2} I_{0}(\Lambda) \sin \left(\Delta+\bar{w}-\bar{w}^{\prime}\right) \\
& +\Lambda \sin \left(\Delta+\bar{w}-\bar{w}^{\prime}\right) \sum_{n=1}^{\infty} I_{n}(\Lambda) \cos \left(n\left(\Delta+\bar{w}-\bar{w}^{\prime}\right)\right) \\
& =\frac{\Lambda}{2} \sin \left(\Delta+\bar{w}-\bar{w}^{\prime}\right) \exp \left(\Lambda \cos \left(\Delta+\bar{w}-\bar{w}^{\prime}\right)\right)
\end{aligned}
$$

(Abramowitz \& Stegun 1968, Sect. 9.6). Furthermore, using the definitions of $\Lambda, \bar{w}$, and $\bar{w}^{\prime}$ I find the relations

$$
\begin{aligned}
& \Lambda \sin \left(\Delta+\bar{\omega}-\bar{\omega}^{\prime}\right)=\frac{\sigma_{u}^{2}}{\kappa^{2}} \\
& \cdot\left\{\frac{\kappa}{2 B} k_{y}^{\prime}\left(k_{x}^{\prime}-k_{x}\right) \cos \Delta+\left(k_{x} k_{x}^{\prime}+\frac{\kappa^{2}}{4 B^{2}} k_{y}^{\prime 2}\right) \sin \Delta\right\}
\end{aligned}
$$

and

$\Lambda \cos \left(\Delta+\bar{\omega}-\bar{\omega}^{\prime}\right)=\frac{\sigma_{u}^{2}}{\kappa^{2}}$

$\times\left\{\left(k_{x} k_{x}^{\prime}+\frac{\kappa^{2}}{4 B^{2}} k_{y}^{\prime 2}\right) \cos \Delta-\frac{\kappa}{2 B} k_{y}^{\prime}\left(k_{x}^{\prime}-k_{x}\right) \sin \Delta\right\}$,

so that the multiple integral (38) can be put into the final form

$$
\begin{aligned}
& -\frac{2 \pi G \Sigma_{0}}{A \kappa\left|k_{y}^{\prime}\right|} \int_{-\infty}^{k_{x}^{\prime}} \mathrm{d} k_{x} \Phi_{\boldsymbol{k}}\left\{\left(k_{x} k_{x}^{\prime}+\frac{\kappa^{2}}{4 B^{2}} k_{y}^{\prime 2}\right) \sin \frac{\kappa\left(k_{x}^{\prime}-k_{x}\right)}{2 A k_{y}^{\prime}}\right. \\
& \left.+\frac{\kappa}{2 B} k_{y}^{\prime}\left(k_{x}^{\prime}-k_{x}\right) \cos \frac{\kappa\left(k_{x}^{\prime}-k_{x}\right)}{2 A k_{y}^{\prime}}\right\} \exp -i \omega \frac{k_{x}^{\prime}-k_{x}}{2 A k_{y}^{\prime}} \\
& \times \exp -\frac{\sigma_{u}^{2}}{\kappa^{2}}\left[\frac{1}{2}\left(k_{x}^{2}+k_{x}^{\prime 2}\right)+\frac{\kappa^{2}}{4 B^{2}} k_{y}^{\prime 2}-\left(k_{x} k_{x}^{\prime}+\frac{\kappa^{2}}{4 B^{2}} k_{y}^{2}\right)\right. \\
& \left.\times \cos \frac{\kappa\left(k_{x}^{\prime}-k_{x}\right)}{2 A k_{y}^{\prime}}+\frac{\kappa}{2 B} k_{y}^{\prime}\left(k_{x}^{\prime}-k_{x}\right) \sin \frac{\kappa\left(k_{x}^{\prime}-k_{x}\right)}{2 A k_{y}^{\prime}}\right] .(65)
\end{aligned}
$$

In expression (65) positive wave numbers $k_{y}^{\prime}$ are assumed. In the case of negative wave numbers $k_{y}^{\prime}$ the integration limits are reversed to $\int_{k_{x}^{\prime}}^{+\infty} \mathrm{d} k_{x}$.

The fundamental integral equation is thus a Volterra equation,

$\Phi_{k^{\prime}}=\int_{-\infty}^{k_{x}^{\prime}} \mathrm{d} k_{x} \mathcal{K}\left(k_{x}, k_{x}^{\prime}\right) \Phi_{k}$

with the kernel

$$
\begin{aligned}
& \mathcal{K}\left(k_{x}, k_{x}^{\prime}\right)=\frac{\pi G \Sigma_{0}}{A \kappa\left|k_{y}^{\prime}\right|} \frac{1}{\sqrt{k_{x}^{\prime 2}+k_{y}^{\prime 2}}} \exp -i \omega \frac{k_{x}^{\prime}-k_{y}}{2 A k_{y}^{\prime}} \\
& \times\left\{\left(k_{x} k_{x}^{\prime}+\frac{\kappa^{2}}{4 B^{2}} k_{y}^{\prime 2}\right) \sin \frac{\kappa\left(k_{x}^{\prime}-k_{x}\right)}{2 A k_{y}^{\prime}}\right. \\
& \left.+\frac{\kappa}{2 B} k_{y}^{\prime}\left(k_{x}^{\prime}-k_{x}\right) \cos \frac{\kappa\left(k_{x}^{\prime}-k_{x}\right)}{2 A k_{y}^{\prime}}\right\} \\
& \times \exp -\frac{\sigma_{u}^{2}}{\kappa^{2}}\left[\frac{1}{2}\left(k_{x}^{2}+k_{x}^{\prime 2}\right)+\frac{\kappa^{2}}{4 B^{2}} k_{y}^{\prime 2}-\left(k_{x} k_{x}^{\prime}+\frac{\kappa^{2}}{4 B^{2}} k_{y}^{\prime 2}\right)\right. \\
& \left.\times \cos \frac{\kappa\left(k_{x}^{\prime}-k_{x}\right)}{2 A k_{y}^{\prime}}+\frac{\kappa}{2 B} k_{y}^{\prime}\left(k_{x}^{\prime}-k_{x}\right) \sin \frac{\kappa\left(k_{x}^{\prime}-k_{x}\right)}{2 A k_{y}^{\prime}}\right] \cdot(67)
\end{aligned}
$$

The integral Eq. (66) is equivalent to that obtained by Julian \& Toomre (1966) although it is derived and presented here in a different way.

\section{Discussion of the Volterra equation}

Surprisingly the Volterra Eq. (66) has no solution. This can be seen by a Fredholm discretization of the equation 
(Morse \& Feshbach 1953). The integral equation is then replaced by a set of algebraic equations with a triangular coefficient matrix. The kernel (67) vanishes on the diagonal, $k_{x}^{\prime}=k_{x}$, so that the diagonal of the coefficient matrix is given by the identity matrix from the left hand side of Eq. (66). The determinant of a triangular matrix is equal to the product of the diagonal elements of the matrix, which is equal to one in this case. This means that the set of algebraic equations and thus the integral equation have no non-trivial solutions. According to the theorem of Fredholm the inhomogeneous Volterra equation

$\Phi_{\boldsymbol{k}^{\prime}}=\int_{-\infty}^{k_{x}^{\prime}} \mathrm{d} k_{x} \mathcal{K}\left(k_{x}, k_{x}^{\prime}\right) \Phi_{\boldsymbol{k}}+r_{\boldsymbol{k}^{\prime}}$

has a unique solution, if the corresponding homogeneous equation has no solution. The solution can be expressed with the resolvent operator $\mathcal{R}$ as

$\Phi_{\boldsymbol{k}^{\prime}}=r_{\boldsymbol{k}^{\prime}}+\int_{-\infty}^{k_{x}^{\prime}} \mathrm{d} k_{x} \mathcal{R}\left(k_{x}, k_{x}^{\prime}\right) r_{\boldsymbol{k}}$

where the resolvent operator is given by a Neumann series,

$\mathcal{R}\left(k_{x}, k_{x}^{\prime}\right)=\sum_{n=1}^{\infty} \mathcal{K}^{(n)}\left(k_{x}, k_{x}^{\prime}\right)$

with

$\mathcal{K}^{(1)}\left(k_{x}, k_{x}^{\prime}\right)=\mathcal{K}\left(k_{x}, k_{x}^{\prime}\right), \quad$ and

$\mathcal{K}^{(n)}\left(k_{x}, k_{x}^{\prime}\right)=\int_{k_{x}}^{k_{x}^{\prime}} \mathrm{d} k_{x}^{\prime \prime} \mathcal{K}\left(k_{x}^{\prime \prime}, k_{x}^{\prime}\right) \mathcal{K}^{(n-1)}\left(k_{x}, k_{x}^{\prime \prime}\right)$.

The inhomogeneity of Eq. (68) represents perturbations of the shearing sheet, which have not been included in the analysis so far. As will be discussed in the next section, these may be due to external forces or massive perturbations within the disk, or any initial non-equilibrium states of the disk. Equation (69) describes then the response of the disk to these perturbations. If they are absent, the shearing sheet does not develop any inhomogeneous spatial structures and perturbations of the velocity field. There is a technical point concerning the functional dependence of the resolvent operator on the frequency $\omega$, which will become important later on. Equation (67) shows that the kernel $\mathcal{K}\left(k_{x}, k_{x}^{\prime}\right)$ is proportional to $\exp -i \omega \frac{k_{x}^{\prime}-k_{x}}{2 A k_{y}^{\prime}}$. This proportionality is maintained under the iterations of Eq. (71), so that the resolvent operator has the same functional dependence as $\mathcal{K}, \mathcal{R}\left(k_{x}, k_{x}^{\prime}\right) \propto \exp -i \omega \frac{k_{x}^{\prime}-k_{x}}{2 A k_{y}^{\prime}}$.

When deriving the Volterra Eq. (68), I have not considered the case $k_{y}^{\prime}=0$, which corresponds in disks with polar geometry to ring-like perturbations. It is straightforward to show using the formalism of Sect. (4) that in this case the disk response to a Fourier component of the general potential perturbation is the corresponding Fourier component of the general disk response, so that they form conjugate pairs for each wave number vector $\boldsymbol{k}^{\prime}=\left(k_{x}^{\prime}, 0\right)$. When self-gravity is taken into account, the result is a dispersion relation $\omega\left(k_{x}\right)$, which is formally identical with that of Lin et al. (1969), although it is valid here only for $k_{y}^{\prime}=0$. Thus the case of $k_{y}^{\prime}=0$ is not generic for cases of even small non-vanishing circumferential wave members. The same holds true for Oort's constant $A$. The dynamical behaviour of a rigidly rotating shearing sheet $(A=0)$ or star disks, in general, is not generic for the behaviour of differentially rotating disks.

\section{Initial value problem}

If the shearing sheet is initially - either at time minus infinity or at some $t=t_{0}-$ not in equilibrium, this must be taken into account explicitely in the Fourier transform of Eq. (20) with respect to the time coordinate. The Laplace transform of the time derivative of the perturbation of the distribution function gives in this case

$$
\begin{aligned}
& \int_{t_{0}}^{\infty} \mathrm{d} t \mathrm{e}^{-i \omega t} \frac{\partial \delta f}{\partial t}=-\delta f\left(t_{0}\right) \mathrm{e}^{-i \omega t_{0}}+\int_{t_{0}}^{\infty} \mathrm{d} t i \omega \mathrm{e}^{-i \omega t} \delta f \\
& =-\delta f\left(t_{0}\right) \mathrm{e}^{-i \omega t_{0}}+i \omega \delta f_{\omega}
\end{aligned}
$$

This means a further inhomogenous term in Eq. (23). Equation (23) is linear, and therefore the solution of the extended equation can be constructed by superposing the solution of Eq. (23) in its original form as derived in Sect. (4) and a solution of

$$
\begin{aligned}
i \omega \delta f_{\omega}+\kappa \frac{\partial \delta f_{\omega}}{\partial w_{1}}+ & \frac{A}{B}\left(J_{2}-\Omega_{0} r_{0}\right) \frac{\partial \delta f_{\omega}}{\partial w_{2}} \\
& -\delta f\left(t_{0}\right) \mathrm{e}^{-i \omega t_{0}}=0 .
\end{aligned}
$$

In this way both inhomogeneous terms of the extended Eq. (23) are taken into account. First, the inhomogenous term of Eq. (73) is Fourier transformed

$$
\begin{aligned}
\delta f\left(t_{0}\right)= & \int_{-\infty}^{+\infty} \mathrm{d} k_{x} \int_{-\infty}^{+\infty} \mathrm{d} k_{y} \delta f_{\boldsymbol{k}}\left(t_{0}\right) \\
& \times \exp \left[i k_{x}\left(\frac{J_{2}-\Omega_{0} r_{0}}{-2 B}+\sqrt{\frac{2 J_{1}}{\kappa}} \sin w_{1}\right)\right. \\
& \left.+i k_{y} w_{2}-i k_{y} \frac{\sqrt{2 \kappa J_{1}}}{2 B} \cos w_{1}\right]
\end{aligned}
$$

Again the background distribution function $f_{0}$ is split off all terms in Eq. (73), in particular $\delta f_{\boldsymbol{k}}\left(t_{0}\right)=f_{0} f_{\boldsymbol{k}}\left(t_{0}\right)$, and a Fourier transform of Eq. (73) with respect to the angle variable $w_{2}$ leads to

$$
\begin{aligned}
& i \omega f_{2, \omega}+\kappa \frac{\mathrm{d} f_{2, \omega}}{\mathrm{d} w_{1}}+i k_{y} \frac{A}{B}\left(J_{2}-\Omega_{0} r_{0}\right) f_{2, \omega} \\
& -\int_{-\infty}^{+\infty} \mathrm{d} k_{x} f_{\boldsymbol{k}}\left(t_{0}\right) \mathrm{e}^{-i \omega t_{0}} \\
& \times \exp i k_{x}\left[\frac{J_{2}-\Omega_{0} r_{0}}{-2 B}+\sqrt{\frac{2 J_{1}}{\kappa}} \sin w_{1}\right] \\
& \times \exp -i k_{y} \frac{\sqrt{2 \kappa J_{1}}}{2 B} \cos w_{1}=0
\end{aligned}
$$

The derivation of the solution of Eq. (75) proceeds in exactly the same way as with Eq. (23) in Sect. (4), so that 
I report only the result using the notation from above

$$
\begin{aligned}
& f_{2, \omega}=-\frac{1}{\kappa} \int_{-\infty}^{+\infty} \mathrm{d} k_{x} f_{\boldsymbol{k}}\left(t_{0}\right) \mathrm{e}^{-i \omega t_{0}} \\
& \times \exp i\left(k_{x} \frac{J_{2}-\Omega_{0} r_{0}}{-2 B}\right) \frac{i}{2 \sin (\pi \eta)} \\
& \times \int_{-\pi}^{\pi} \mathrm{d} w_{1}^{\prime} \exp i\left[\eta w_{1}^{\prime}-\xi \sin \left(w_{1}^{\prime}+w_{1}-\bar{w}\right)\right] .
\end{aligned}
$$

The disk response to the potential perturbation is now given by

$\delta f_{\omega}=f_{0}\left(f_{1, \omega}+f_{2, \omega}\right) \exp i\left(\omega t+k_{y} w_{2}\right)$.

The second part of the disk response has also to be considered in the Poisson equation. Again this can be done separately and the expression analogous to (38) is given by

$$
\begin{aligned}
& -\frac{4 \pi G}{(2 \pi)^{2}} \int_{0}^{2 \pi} \mathrm{d} w_{1} \int_{-\infty}^{+\infty} \mathrm{d} w_{2} \int_{0}^{\infty} \mathrm{d} J_{1} \int_{-\infty}^{+\infty} \mathrm{d} J_{2} \frac{\kappa}{-2 B} \\
& \times \frac{\Sigma_{0}}{2 \pi \sigma_{u}^{2}} \exp \left(-\frac{\kappa}{\sigma_{u}^{2}} J_{1}\right) \\
& \times \int_{-\infty}^{+\infty} \mathrm{d} k_{y} \exp \left(i k_{y} w_{2}\right) \frac{1}{\kappa} \int_{-\infty}^{+\infty} \mathrm{d} k_{x} f_{\boldsymbol{k}}\left(t_{0}\right) \mathrm{e}^{-i \omega t_{0}} \\
& \times \exp \left(i k_{x} \frac{J_{2}-\Omega_{0} r_{0}}{-2 B}\right) \frac{i}{2 \sin (\pi \eta)} \\
& \times \int_{-\pi}^{+\pi} \mathrm{d} w_{1}^{\prime} \exp i\left[\eta w_{1}^{\prime}-\xi \sin \left(w_{1}^{\prime}+w_{1}-\bar{w}\right)\right] \\
& \times \exp -i\left[k_{x}^{\prime} \frac{J_{2}-\Omega_{0} r_{0}}{-2 B}+k_{x}^{\prime} \sqrt{\frac{2 J_{1}}{\kappa}} \sin w_{1}\right] \\
& \times \exp i\left[k_{y}^{\prime} w_{2}-k_{y}^{\prime} \frac{\sqrt{2 \kappa J_{1}}}{2 B} \cos w_{1}\right] .
\end{aligned}
$$

The quadratures can be carried out in the same way as in Sect. (6) and I report again only the result,

$$
\begin{aligned}
& \frac{2 \pi G \Sigma_{0}}{A\left|k_{y}^{\prime}\right|} \int_{-\infty}^{k_{x}^{\prime}} \mathrm{d} k_{x} f_{k_{x}, k_{y}^{\prime}}\left(t_{0}\right) \mathrm{e}^{-i \omega t_{0}} \exp -i \omega \frac{k_{x}^{\prime}-k_{x}}{2 A k_{y}^{\prime}} \\
& \times \exp -\frac{\sigma_{u}^{2}}{\kappa^{2}}\left[\frac{1}{2}\left(k_{x}^{2}+\frac{\kappa^{2}}{4 B^{2}} k_{y}^{\prime 2}\right)+\frac{1}{2}\left(k_{x}^{\prime 2}+\frac{\kappa^{2}}{4 B^{2}} k_{y}^{\prime 2}\right)\right. \\
& -\left(k_{x}^{\prime} k_{x}+\frac{\kappa^{2}}{4 B^{2}} k_{y}^{\prime 2}\right) \cos \frac{\kappa\left(k_{x}^{\prime}-k_{x}\right)}{2 A k_{y}^{\prime}} \\
& \left.+\frac{\kappa}{2 B} k_{y}^{\prime}\left(k_{x}^{\prime}-k_{x}\right) \sin \frac{\kappa\left(k_{x}^{\prime}-k_{x}\right)}{2 A k_{y}^{\prime}}\right],
\end{aligned}
$$

where positive wave numbers $k_{y}^{\prime}$ are assumed. In the case of negative wave numbers $k_{y}^{\prime}$ the integration limits have to be reversed to $\int_{k_{x}^{\prime}}^{\infty} \mathrm{d} k_{x}$. Thus the inhomogenous term of the integral Eq. (68) can be written as

$r_{\boldsymbol{k}^{\prime}}=\int_{-\infty}^{k_{x}^{\prime}} \mathrm{d} k_{x} \mathcal{L}\left(k_{x}, k_{x}^{\prime}\right) f_{k_{x}, k_{x}^{\prime}}\left(t_{0}\right) \mathrm{e}^{-i \omega t_{0}}$ with

$$
\begin{aligned}
& \mathcal{L}\left(k_{x}, k_{x}^{\prime}\right)=-\frac{\pi G \Sigma_{0}}{A\left|k_{y}^{\prime}\right|} \frac{1}{\sqrt{k_{x}^{\prime 2}+k_{y}^{\prime 2}}} \exp -i \omega \frac{k_{x}^{\prime}-k_{x}}{2 A k_{y}^{\prime}} \\
& \times \exp -\frac{\sigma_{u}^{2}}{\kappa^{2}}\left[\frac{1}{2}\left(k_{x}^{2}+\frac{\kappa^{2}}{4 B^{2}} k_{y}^{\prime 2}\right)+\frac{1}{2}\left(k_{x}^{\prime 2}+\frac{\kappa^{2}}{4 B^{2}} k_{y}^{\prime 2}\right)\right. \\
& -\left(k_{x}^{\prime} k_{x}+\frac{\kappa^{2}}{4 B^{2}} k_{y}^{\prime 2}\right) \cos \frac{\kappa\left(k_{x}^{\prime}-k_{x}\right)}{2 A k_{y}^{\prime}} \\
& \left.+\frac{\kappa}{2 B} k_{y}^{\prime}\left(k_{x}^{\prime}-k_{x}\right) \sin \frac{\kappa\left(k_{x}^{\prime}-k_{x}\right)}{2 A k_{y}^{\prime}}\right],
\end{aligned}
$$

where the $\omega$-dependent term has exactly the same form as in the kernel function $\mathcal{K}$. The general solution of the integral Eq. (68) has then the specific form

$$
\begin{aligned}
& \Phi_{\boldsymbol{k}^{\prime}}=\int_{-\infty}^{k_{x}^{\prime}} \mathrm{d} k_{x} \mathcal{L}\left(k_{x}, k_{x}^{\prime}\right) f_{k_{x}, k_{x}^{\prime}}\left(t_{0}\right) \mathrm{e}^{-i \omega t_{0}} \\
& +\int_{-\infty}^{k_{x}^{\prime}} \mathrm{d} k_{x} \mathcal{R}\left(k_{x}, k_{x}^{\prime}\right) \int_{-\infty}^{k_{x}} \mathrm{~d} k_{x}^{\prime \prime} \mathcal{L}\left(k_{x}^{\prime \prime}, k_{x}\right) f_{k_{x}^{\prime \prime}, k_{y}^{\prime}}\left(t_{0}\right) \mathrm{e}^{-i \omega t_{0}} .
\end{aligned}
$$

The potential (82) is still defined in Fourier space, but, when transformed back to time and spatial coordinates, gives the description of the evolution of the disturbance of the shearing sheet. Illustrative examples are described in the next section.

It is instructive to consider the behaviour of the solution (82) in the limit $t \rightarrow \infty\left(k_{y}^{\prime}>0\right)$,

$$
\begin{aligned}
& \lim _{t \rightarrow \infty} \frac{1}{2 \pi} \int_{-\infty}^{+\infty} \mathrm{d} \omega \mathrm{e}^{i \omega t} \Phi_{\boldsymbol{k}^{\prime}, \omega} \\
& =\lim _{t \rightarrow \infty} \int_{-\infty}^{k_{x}^{\prime}} \mathrm{d} k_{x} f_{k_{x}, k_{y}^{\prime}}\left(t_{0}\right) \\
& \times 2 A k_{y}^{\prime} \delta\left(k_{x}-k_{x}^{\prime}+2 A k_{y}^{\prime}\left(t-t_{0}\right)\right) \tilde{\mathcal{L}}\left(k_{x}, k_{x}^{\prime}\right) \\
& +\lim _{t \rightarrow \infty} \int_{-\infty}^{k_{x}^{\prime}} \mathrm{d} k_{x} \tilde{\mathcal{R}}\left(k_{x}, k_{x}^{\prime}\right) \int_{-\infty}^{k_{x}} \mathrm{~d} k_{x}^{\prime \prime} \tilde{\mathcal{L}}\left(k_{x}^{\prime \prime}, k_{x}\right) f_{k_{x}^{\prime \prime}, k_{y}^{\prime}}\left(t_{0}\right) \\
& \times 2 A k_{y}^{\prime} \delta\left(k_{x}^{\prime \prime}-k_{x}^{\prime}+2 A k_{y}^{\prime}\left(t-t_{0}\right)\right) \\
& =\lim _{t \rightarrow \infty} 2 A k_{y}^{\prime} \tilde{\mathcal{L}}\left(k_{x}^{\prime}-2 A k_{y}^{\prime}\left(t-t_{0}\right), k_{x}^{\prime}\right) \\
& \times f_{k_{x}^{\prime}-2 A k_{y}^{\prime}\left(t-t_{0}\right), k_{y}^{\prime}}\left(t_{0}\right) \\
& +\lim _{t \rightarrow \infty} 2 A k_{y}^{\prime} \int_{-\infty}^{k_{x}^{\prime}} \mathrm{d} k_{x} \tilde{\mathcal{R}}\left(k_{x}, k_{x}^{\prime}\right) \tilde{\mathcal{L}}\left(k_{x}^{\prime}-2 A k_{y}^{\prime}\left(t-t_{0}\right), k_{x}\right) \\
& \times f_{k_{x}^{\prime}-2 A k_{y}^{\prime}\left(t-t_{0}\right), k_{y}^{\prime}}\left(t_{0}\right),
\end{aligned}
$$

where operators with an overhead tilde denote again operators with the $\omega$-dependent terms (cf. Sect. 7 and Eq. (81)) split off. As can be seen from Eq. (81) the $\tilde{\mathcal{L}}$ functions decay as

$$
\begin{aligned}
& \lim _{t \rightarrow \infty} \tilde{\mathcal{L}}\left(k_{x}^{\prime}-2 A k_{y}^{\prime}\left(t-t_{0}\right), k_{x}^{\prime}\right) \\
& =\lim _{t \rightarrow \infty} \tilde{\mathcal{L}}\left(k_{x}^{\prime}-2 A k_{y}^{\prime}\left(t-t_{0}\right), k_{x}\right) \\
& \propto \lim _{t \rightarrow \infty} \exp -\frac{\sigma_{u}^{2}}{2 \kappa^{2}}\left(2 A k_{y}^{\prime}\left(t-t_{0}\right)\right)^{2}=0
\end{aligned}
$$


which means that the perturbation will die out eventually. This was first noted by Julian \& Toomre (1966). The physical reason is that the effective radial wave length of the perturbation, $\pi /\left(A k_{y}^{\prime}\left(t-t_{0}\right)\right)$, becomes much smaller than the typical epicycle size of the stellar orbits, measured by $\sigma_{u} / \kappa$, so that the disk can no longer sustain such a perturbation. This is not the case for gas disks (Goldreich \& Tremaine 1978).

\section{Illustrative examples}

The characteristics of the solutions of the Volterra integral Eq. (68) become immediately clear, when the shearing sheet is assumed to be perturbed initially by a single sinusoidal wave with a wave number $\boldsymbol{k}^{\text {in }}\left(k_{y}^{\text {in }}>0\right)$,

$\delta f_{\boldsymbol{k}}\left(t_{0}=0\right)=f_{k_{y}^{\text {in }}}^{\text {in }} \delta\left(k_{x}-k_{x}^{\text {in }}\right)$.

According to Eq. (80) this implies an inhomogeneity of the Volterra equation

$r_{k_{x}^{\prime}, k_{y}^{\text {in }}}=f_{k_{y}^{\text {in }}}^{\text {in }} \mathcal{L}\left(k_{x}^{\text {in }}, k_{x}^{\prime}\right)$,

and the solution of the Volterra equation is given according to Eq. (82) by

$$
\begin{aligned}
& \Phi_{k_{x}^{\prime}, k_{y}^{\text {in }}}=f_{k_{y}^{\text {in }}}^{\text {in }} \mathcal{L}\left(k_{x}^{\text {in }}, k_{x}^{\prime}\right) \\
& +\int_{k_{x}^{\text {in }}}^{k_{x}^{\prime}} \mathrm{d} k_{x} \mathcal{R}\left(k_{x}, k_{x}^{\prime}\right) f_{k_{y}^{\text {in }}}^{\text {in }} \mathcal{L}\left(k_{x}^{\text {in }}, k_{x}\right) .
\end{aligned}
$$

Transforming this back to time and spatial coordinates leads to

$$
\begin{aligned}
& \Phi_{k_{x}^{\prime}, k_{y}^{\text {in }}, t}=\frac{1}{2 \pi} \int_{-\infty}^{+\infty} \mathrm{d} \omega \mathrm{e}^{i \omega t} \Phi_{k_{x}^{\prime}, k_{y}^{\text {in }}, \omega} \\
& =f_{k_{y}^{\text {in }}}^{\text {in }} \tilde{\mathcal{L}}\left(k_{x}^{\text {in }}, k_{x}^{\prime}\right) \delta\left(t+\frac{k_{x}^{\text {in }}-k_{x}^{\prime}}{2 A k_{y}^{\text {in }}}\right) \\
& +f_{k_{y}^{\text {in }}}^{\text {in }} \int_{k_{x}^{\text {in }}}^{k_{x}^{\prime}} \mathrm{d} k_{x} \tilde{\mathcal{R}}\left(k_{x}, k_{x}^{\prime}\right) \tilde{\mathcal{L}}\left(k_{x}^{\text {in }}, k_{x}\right) \delta\left(t+\frac{k_{x}^{\text {in }}-k_{x}^{\prime}}{2 A k_{y}^{\text {in }}}\right)
\end{aligned}
$$

and then

$$
\begin{aligned}
& \delta \Phi(x, y, t)=\frac{1}{2 \pi} \int_{-\infty}^{+\infty} \mathrm{d} k_{x}^{\prime} \mathrm{e}^{i\left[k_{x}^{\prime} x+k_{y}^{\text {in }} y\right]} \Phi_{k_{x}^{\prime}, k_{y}^{\text {in }}}(t) \\
& =\frac{A k_{y}^{\text {in }}}{\pi} f_{k_{y}^{\text {in }}}^{\text {in }}\left\{\tilde{\mathcal{L}}\left(k_{x}^{\text {in }}, k_{x}^{\text {in }}+2 A k_{y}^{\text {in }} t\right)\right. \\
& \left.+\int_{k_{x}^{\text {in }}}^{k_{x}^{\text {in }}+2 A k_{y}^{\text {in }} t} \mathrm{~d} k_{x} \tilde{\mathcal{R}}\left(k_{x}, k_{x}^{\text {in }}+2 A k_{y}^{\text {in }} t\right) \tilde{\mathcal{L}}\left(k_{y}^{\text {in }}, k_{x}\right)\right\} \\
& \times \exp i\left[\left(k_{x}^{\text {in }}+2 A k_{y}^{\text {in }} t\right) x+k_{y}^{\text {in }} y\right],
\end{aligned}
$$

where the overhead tildes mean again that the $\omega$ dependent terms have been split off the operators $\mathcal{L}$ and $\mathcal{R}$ (cf. the remarks at the end of Sect. 7). An analogous expression can be derived for the surface density of the perturbation $\delta \Sigma$. Inserting (cf. Eq. 34),

$\Sigma_{\boldsymbol{k}}=-\frac{\sqrt{k_{x}^{2}+k_{y}^{2}}}{2 \pi G} \Phi_{\boldsymbol{k}}$, into Eq. (89) gives immediately

$$
\begin{aligned}
& \delta \Sigma(x, y, t)=-\frac{1}{2 \pi G} \frac{A k_{y}^{\text {in }}}{\pi} f_{k_{y}^{\text {in }}}^{\text {in }} \sqrt{\left(k_{x}^{\text {in }}+2 A k_{y}^{\text {in }} t\right)^{2}+k_{y}^{\text {in }^{2}}} \\
& \times\left\{\tilde{\mathcal{L}}\left(k_{x}^{\text {in }}, k_{x}^{\text {in }}+2 A k_{y}^{\text {in }} t\right)+\int_{k_{x}^{\text {in }}}^{k_{x}^{\text {in }}+2 A k_{y}^{\text {in }} t} \mathrm{~d} k_{x}\right. \\
& \left.\times \tilde{\mathcal{R}}\left(k_{x}^{\text {in }}, k_{x}^{\text {in }}+2 A k_{y}^{\text {in }} t\right) \tilde{\mathcal{L}}\left(k_{x}^{\text {in }}, k_{x}\right)\right\} \\
& \times \exp i\left[\left(k_{x}^{\text {in }}+2 A k_{y}^{\text {in }} t\right) x+k_{y}^{\text {in }} y\right] .
\end{aligned}
$$

The spatial pattern described by Eq. (91) is still a sinusoidal wave, however with shearing wave crests. The wave crests, which are oriented perpendicular to the wave number vector, are defined by lines

$\left(k_{x}^{\text {in }}+2 A k_{y}^{\text {in }} t\right) x+k_{y}^{\text {in }} y=$ const.,

which tilt at the rate

$\frac{\mathrm{d}}{\mathrm{d} t}\left(\frac{\Delta y}{\Delta x}\right)=2 A$.

This corresponds exactly to the linear shear flow (11) of the shearing sheet. The amplitude function in Eq. (91) describes the growth and damping of the perturbation. As will be illustrated below, the amplitude function shows also some oscillatory behaviour, so that the solutions of the Volterra equation have also to some extent the characteristics of moving wave trains. For this complex phenomenon Toomre (1981) has coined the term "swingamplification".

Unfortunately the amplitude function in Eq. (91) cannot be calculated analytically. However it needs only very modest numerical work to solve the original Volterra equation by Fredholm discretization numerically. The coefficient matrix of the resulting set of algebraic equations is already in triangular form and extremely well behaved, because the kernel function (67) falls off in both the $k_{x}$ and $k_{x}^{\prime}$ directions, respectively, tapered by gaussians. The Volterra equation can be written in dimensionless form, if the wave numbers are measured in units of the critical wave number,

$k_{\text {crit }}=\frac{\kappa^{2}}{2 \pi G \Sigma_{0}}$,

and the radial velocity dispersion $\sigma_{u}$ is expressed in terms of the Toomre stability parameter $Q$

$Q=\frac{\kappa \sigma_{u}}{3.36 G \Sigma_{0}}$

The numerical coefficient in the argument of the exponential function of the kernel (67) is then given by

$\frac{\sigma_{u}^{2}}{\kappa^{2}} k_{\mathrm{crit}}^{2}=0.2856 Q^{2}$.

Thus the kernel $\mathcal{K}$ and the $\mathcal{L}$ function are parameterized by the values of $A / \Omega_{0}$ and $Q$. The numerical scheme to solve the integral equation is briefly described in the Appendix. 


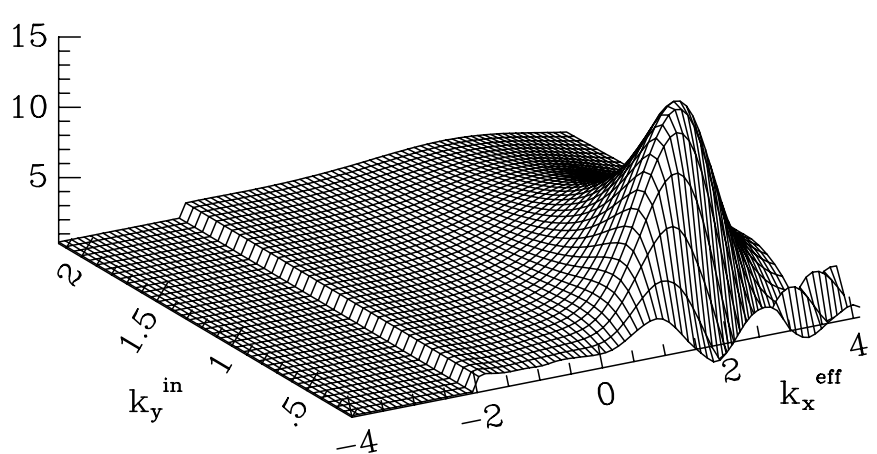

Fig. 1. Amplification of shearing density waves. $k_{y}^{\text {in }}$ denotes the - constant - circumferential wave numbers in units of $k_{\text {crit }}$. $k_{x}^{\text {eff }}$ denotes the effective radial wave number and is given by $k_{x}^{\text {in }}+2 A k_{y}^{\text {in }} t$, so that it increases from left to right during a swing-amplification event. The initial radial wave number is chosen as $k_{x}^{\text {in }}=-2 k_{\text {crit }}$, which corresponds to initially leading arms. The parameters of the disk model are $A / \Omega_{0}=0.5$ and $Q=1.4$

In Fig. 1 the amplitude function of Eq. (91) is shown for the case of $A / \Omega_{0}=0.5$ and $Q=1.4$. The amplification factors are drawn as function of $k_{x}^{\text {eff }}=k_{x}^{\text {in }}+2 A k_{y}^{\text {in }} t$ abscissae for increasing circumferential wave numbers $k_{y}^{\text {in }}$. The sinusoidal waves have been incited with unit amplitude and with an initial radial wave number $k_{x}^{\text {in }}=-2 k_{\text {crit }}$. As can be seen from Fig. 1 maximum amplification is achieved, if the circumferential wave number is about $0.5 k_{\text {crit }}$. This is the exact equivalent of the " $X=2$ " criterion of Toomre (1981). The maximum of amplification is reached, when the effective radial wave number is $k_{x}^{\text {eff }} \approx 2 k_{\text {crit }}$, which corresponds to fairly open trailing "spiral" arms. The duration of the amplification event is given by

$t=\frac{k_{x}^{\mathrm{eff}}-k_{x}^{\mathrm{in}}}{2 A k_{x}^{\mathrm{in}}}$,

and is thus of the order of an orbital period $2 \pi / \Omega_{0}$. It can be also seen from Fig. 1 that, if the circumferential wave number $k_{y}^{\text {in }}$ becomes very small, which corresponds to tightly wound "spiral" arms, the duration of amplification is fairly long and approaches the asymptotic WKB limit (Lin et al. 1969).

While in Fig. 1 the arms were initially leading, in Fig. 2 the amplification of initially trailing arms is shown. As can be seen from Fig. 2 there is hardly any amplification, which illustrates the well known result (Toomre 1981) that the swing-amplification mechanism requires leading input in order to amplify density waves.

\section{Parameter study}

The solutions of the Volterra equation are parametrized by the values of $A / \Omega_{0}$ and $Q$. The peak of maximum amplification in wave number space shifts, when Oort's constant $A$ is varied. I have calculated a grid of models with varying values of $A / \Omega_{0}$ keeping the Toomre parameter constant at $Q=1.4$ and have determined for each model the circumferential wave numbers for which peak amplification

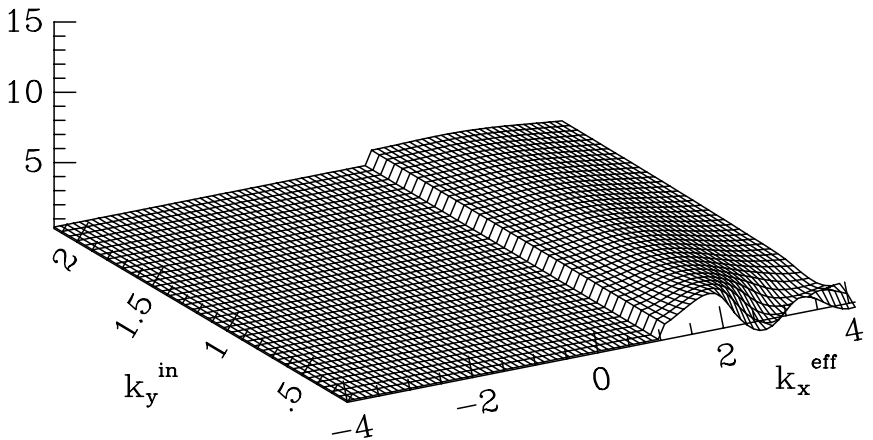

Fig. 2. Same as in Fig. 1, but the initial radial wave number is chosen as $k_{x}^{\text {in }}=+1 k_{\text {crit }}$. The parameters of the disk model are again $A / \Omega_{0}=0.5$ and $Q=1.4$

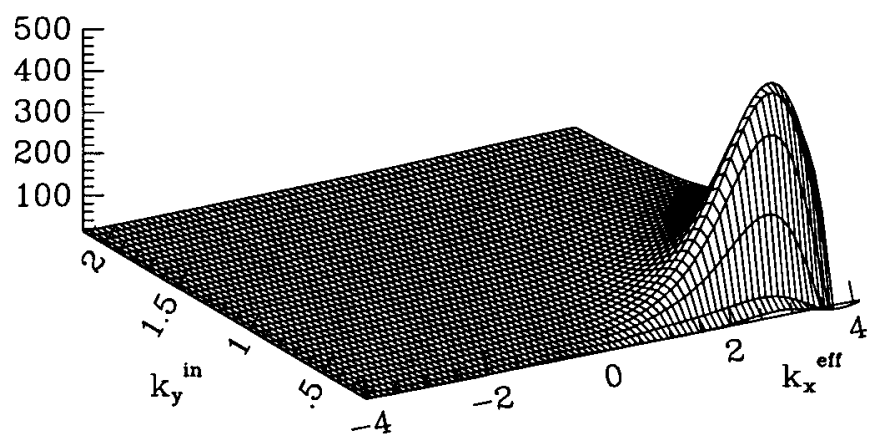

Fig. 3. Same as in Fig. 1, but the parameters of the disk model have been changed to $A / \Omega_{0}=0.5$ and $Q=1$

is reached. These can be fitted empirically by a relation of the form

$\frac{k_{y, \max }}{k_{\mathrm{crit}}}=1.932-5.186\left(\frac{A}{\Omega_{0}}\right)+4.704\left(\frac{A}{\Omega_{0}}\right)^{2}$

over the range $0.1 \leq\left(\frac{A}{\Omega_{0}}\right) \leq 0.5$, which corresponds to rising rotation curves. This domain is particularly relevant, because in spiral galaxies the optically visible star disks often extend only over regions, where the rotation curves are still rising. Relation (98) is in perfect agreement with the result of Athanassoula (1984), who sudied the influence of the shape of the rotation curve on swingamplification by a different approach.

The amplification factor itself depends strongly on the value of the Toomre stability parameter. In Figs. 3 and 4 amplitude functions are shown which correspond to $Q=$ 1 and $Q=2$, respectively. As can be seen from Fig. 3 amplification becomes very high, when $Q$ approaches the limit of Jeans instability $Q=1$, while in the case $Q=2$ the disk is dynamically too hot to develop any structure.

\section{External perturbations}

When the shearing sheet is subjected to an external potential perturbation $\Phi^{\text {ext }}$, the sheet will respond to it and the response generates its own gravitational potential $\Phi^{\mathrm{int}}$. The overall gravitational potential is obviously given by

$\Phi=\Phi^{\mathrm{int}}+\Phi^{\mathrm{ext}}$ 


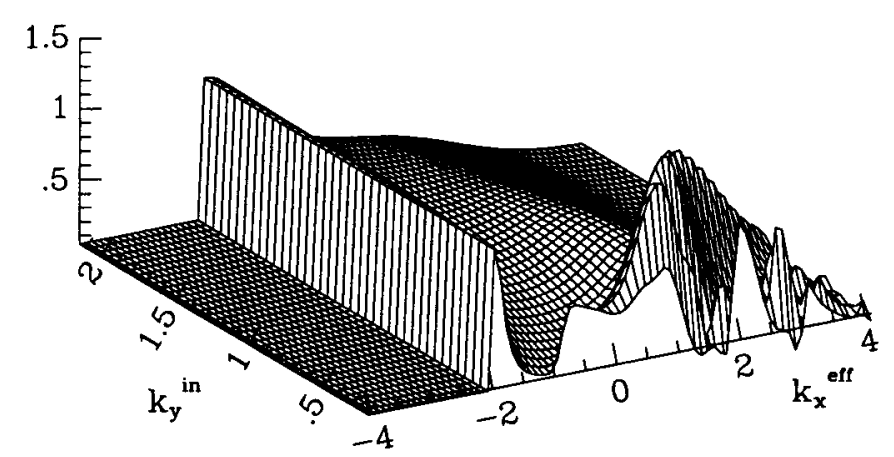

Fig. 4. Same as in Fig. 1, but the parameters of the disk model have been changed to $A / \Omega_{0}=0.5$ and $Q=2$

This has to be inserted into the Boltzmann equation, which leads to the determination of the distribution function of the perturbed sheet and eventually to the right hand side of Eq. (35). Into the left hand side of Eq. (35), however, only the gravitational potential generated by the perturbed sheet, $\Phi^{\text {int }}=\Phi-\Phi^{\text {ext }}$, has to be inserted. If the external potential perturbation is Fourier transformed with respect to time and spatial coordinates, its contribution to the left hand side of Eq. (35) can be calculated using Eq. (36) leading to an inhomogeneity of the Volterra Eq. (68)

$r_{\boldsymbol{k}^{\prime}}=\Phi_{\boldsymbol{k}^{\prime}, \omega}^{\mathrm{ext}}$.

\subsection{Excitation of a density wave}

If the shearing sheet is perturbated by an impulse of the form

$\Phi^{\mathrm{ext}}=\Phi_{0} \exp \left\{i\left[k_{x}^{\mathrm{in}} x+k_{y}^{\mathrm{in}} y\right]-\frac{1}{2}\left(\frac{t}{T}\right)^{2}\right\}$,

where the time constant $T$ indicates the length of its duration, the sheet responds with a swing amplified density wave. The Fourier transform of Eq. (101) with respect to time and spatial coordinates leads to an inhomogeneity of the Volterra Eq. (68) of the form

$r_{\boldsymbol{k}^{\prime}}=(2 \pi)^{\frac{5}{2}} \Phi_{0} T \delta\left(k_{x}^{\prime}-k_{x}^{\text {in }}\right) \delta\left(k_{y}^{\prime}-k_{y}^{\text {in }}\right) \mathrm{e}^{-\frac{1}{2} \omega^{2} T^{2}}$.

The solution of the Volterra equation is given according to Eq. (69) by

$$
\begin{aligned}
& \Phi_{\boldsymbol{k}^{\prime}}=(2 \pi)^{\frac{5}{2}} \Phi_{0} T \delta\left(k_{x}^{\prime}-k_{x}^{\text {in }}\right) \delta\left(k_{y}^{\prime}-k_{y}^{\text {in }}\right) \mathrm{e}^{-\frac{1}{2} \omega^{2} T^{2}} \\
& +\int_{-\infty}^{k_{x}^{\prime}} \mathrm{d} k_{x} \mathcal{R}\left(k_{x}, k_{x}^{\prime}\right)(2 \pi)^{\frac{5}{2}} \Phi_{0} T \delta\left(k_{x}-k_{x}^{\text {in }}\right) \delta\left(k_{y}^{\prime}-k_{y}^{\text {in }}\right) \\
& \times \mathrm{e}^{-\frac{1}{2} \omega^{2} T^{2}} .
\end{aligned}
$$

Transforming this back to time and spatial coordinates leads to

$$
\begin{aligned}
& \Phi(x, y, t)=\Phi_{0} \exp \left\{i\left[k_{x}^{\mathrm{in}} x+k_{y}^{\mathrm{in}} y\right]-\frac{1}{2}\left(\frac{t}{T}\right)^{2}\right\} \\
& +\frac{1}{2 \pi} \int_{-\infty}^{+\infty} \mathrm{d} k_{x}^{\prime} \mathrm{e}^{i\left[k_{x}^{\prime} x+k_{y}^{\mathrm{in}} y\right]} \int_{-\infty}^{k_{x}^{\prime}} \mathrm{d} k_{\mathrm{x}} \tilde{\mathcal{R}}\left(k_{x}, k_{x}^{\prime}\right)
\end{aligned}
$$

$$
\begin{aligned}
& \times \frac{1}{2 \pi} \int_{-\infty}^{+\infty} \mathrm{d} \omega \mathrm{e}^{i \omega\left(t-\frac{k_{x}^{\prime}-k_{x}}{2 A k_{y}^{\text {in }}}\right)} \cdot(2 \pi)^{\frac{5}{2}} \Phi_{0} T \delta\left(k_{x}-k_{x}^{\text {in }}\right) \\
& \times \mathrm{e}^{-\frac{1}{2} \omega^{2} T^{2}}
\end{aligned}
$$

where the $\omega$-dependent term of the resolvent operator $\mathcal{R}$ has been written separately (cf. Sect. 7). Carrying out the integrations gives

$$
\begin{aligned}
& \Phi(x, y, t)=\Phi_{0} \exp \left\{i\left[k_{x}^{\text {in }} x+k_{y}^{\text {in }} y\right]-\frac{1}{2}\left(\frac{t}{T}\right)^{2}\right\} \\
& +\Phi_{0} \int_{k_{x}^{\text {in }}}^{+\infty} \mathrm{d} k_{x}^{\prime} \mathrm{e}^{i\left[k_{x}^{\prime} x+k_{y}^{\text {in }} y\right]} \tilde{\mathcal{R}}\left(k_{x}^{\text {in }}, k_{x}^{\prime}\right) \\
& \times \exp -\frac{1}{2 T^{2}}\left(t+\frac{k_{x}^{\text {in }}-k_{x}^{\prime}}{2 A k_{y}^{\text {in }}}\right)^{2} .
\end{aligned}
$$

If the time constant is small, Eq. (105) can be approximately written as

$$
\begin{aligned}
& \Phi(x, y, t) \approx \Phi_{0} \exp \left\{i\left[k_{x}^{\mathrm{in}} x+k_{y}^{\mathrm{in}} y\right]-\frac{1}{2}\left(\frac{t}{T}\right)^{2}\right\} \\
& +\widehat{\Phi_{0}} \exp i\left[\left(k_{x}^{\mathrm{in}}+2 A k_{y}^{\mathrm{in}} t\right) x+k_{y}^{\mathrm{in}} y\right] \\
& \times \tilde{\mathcal{R}}\left(k_{x}^{\mathrm{in}}, k_{x}^{\mathrm{in}}+2 A k_{y}^{\mathrm{in}} t\right) .
\end{aligned}
$$

The first term in Eq. (105) represents obviously the initial impulse, whereas the second term describes a swing amplified density wave exactly of the kind already found in the treatment of the initial value problem in Sect. (9) (cf. Eq. (89)).

\subsection{Response to a point mass}

The gravitational potential of a point mass resting at the origin of the shearing sheet is given by

$\Phi^{\mathrm{ext}}=-\left.\frac{G m}{r}\right|_{z=0}=-\frac{G m}{\sqrt{x^{2}+y^{2}}}$.

Its Fourier transform with respect to spatial coordinates can be calculated using formulae (3.754) and (6.671) of Gradshteyn \& Ryzhik (1965) giving

$\Phi_{\boldsymbol{k}^{\prime}}^{\mathrm{ext}}=-\frac{2 \pi G m}{\sqrt{k_{x}^{\prime 2}+k_{y}^{\prime 2}}}$

so that the inhomogeneity of the Volterra equation has the form

$r_{\boldsymbol{k}^{\prime}}=-\frac{4 \pi^{2} G m}{\sqrt{k_{x}^{\prime 2}+k_{y}^{\prime 2}}} \delta(\omega)$.

According to Eq. (69) the gravitational of the potential of the perturbation is then

$$
\begin{aligned}
& \Phi_{\boldsymbol{k}^{\prime}}=-\frac{4 \pi^{2} G m}{\sqrt{k_{x}^{\prime 2}+k_{y}^{\prime 2}}} \delta(\omega) \\
& -\int_{-\infty}^{k_{x}^{\prime}} \mathrm{d} k_{x} \mathcal{R}\left(k_{x}, k_{x}^{\prime}\right) \frac{4 \pi^{2} G m}{\sqrt{k_{x}^{\prime 2}+k_{y}^{\prime 2}}} \delta(\omega),
\end{aligned}
$$


and the corresponding surface density distribution (cf. Sect. 9)

$\Sigma_{\boldsymbol{k}^{\prime}}=2 \pi m \delta(\omega)$

$+\sqrt{k_{x}^{\prime 2}+k_{y}^{\prime 2}} \int_{-\infty}^{k_{x}^{\prime}} \mathrm{d} k_{x} \mathcal{R}\left(k_{x}, k_{x}^{\prime}\right) \frac{2 \pi m \delta(\omega)}{\sqrt{k_{x}^{2}+k_{y}^{\prime 2}}} \cdot$

Transforming this back to time and spatial coordinates leads to

$\Sigma(x, y, t)=m \delta(x) \delta(y)+\frac{m}{4 \pi^{2}} \int_{-\infty}^{+\infty} \mathrm{d} k_{x}^{\prime} \int_{-\infty}^{+\infty} \mathrm{d} k_{y}^{\prime}$

$\int_{-\infty}^{k_{x}^{\prime}} \mathrm{d} k_{x} \frac{\sqrt{k_{x}^{\prime 2}+k_{y}^{\prime 2}}}{\sqrt{k_{x}^{2}+k_{y}^{\prime 2}}} \tilde{\mathcal{R}}\left(k_{x}, k_{x}^{\prime}\right) \mathrm{e}^{i\left[k_{x}^{\prime} x+k_{y}^{\prime} y\right]}$.

The first term of Eq. (112) represents obviously the imposed point mass, whereas the second term describes a stationary polarization cloud induced by the point mass. Exactly the same polarization clouds have been found by Julian \& Toomre (1966) or Toomre \& Kalnajs (1991). Figure 5 shows a contour diagram of the surface density distribution of such a polarization cloud calculated numerically by solving the Volterra equation with an inhomogeneity according to Eq. (109) by Fredholm discretization. I find the same surprisingly high surface density of the polarization cloud as Julian \& Toomre (1966). In the example shown in Fig. 5 the effective mass of the imposed perturber is increased by a factor of about ten due to the response of the disk.

\subsection{Passage of a point mass}

The dynamical effect of a satellite galaxy encountering a galactic disk on an unbound coplanar orbit can be simulated in the shearing sheet by considering a point mass moving at constant speed $v_{0}$ along the $y$-axis. The external potential perturbation is then given by

$\Phi^{\mathrm{ext}}=-\frac{G m}{\sqrt{x^{2}+\left(y-v_{0} t\right)^{2}}} \cdot$

Using again the formulae (3.754) and (6.671) of Gradshteyn \& Ryzhik (1965) the potential perturbation can be Fourier transformed as

$\Phi_{\boldsymbol{k}^{\prime}}^{\mathrm{ext}}=-\frac{2 \pi G m}{\sqrt{k_{x}^{\prime 2}+k_{y}^{\prime 2}}} \mathrm{e}^{-i k_{y}^{\prime} v_{0} t}$

leading to an inhomogeneity of the Volterra equation of the form

$r_{\boldsymbol{k}^{\prime}}=-\frac{4 \pi^{2} G m}{\sqrt{k_{x}^{\prime 2}+k_{y}^{\prime 2}}} \delta\left(\omega+k_{y}^{\prime} v_{0}\right)$.

The solution of the Volterra equation has then an analogous form to Eqs. (110) and (111). Transforming this back

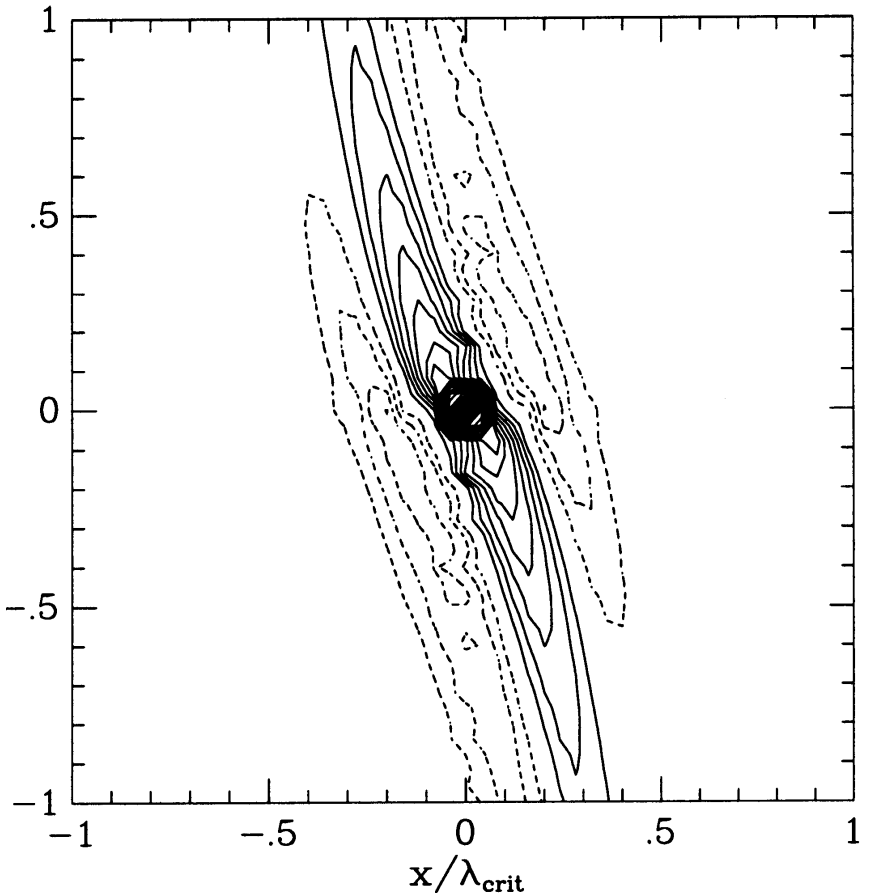

Fig. 5. Response of the shearing sheet to an imposed point mass illustrated by a contour diagram of the surface density distribution. The inner peak represents the delta-function like point mass spread out according to the finite spatial resolution of $2 \pi / k_{\max }=\lambda_{\text {crit }} / 8$ (FWHM). Contour levels at multiples of $25 \mathrm{~m} / \lambda_{\text {crit }}^{2}$ are shown, where $m$ denotes the imposed point mass. Negative density perturbations are drawn as dashed lines

to time and spatial coordinates leads to

$$
\begin{aligned}
& \Sigma(x, y, t)=m \delta(x) \delta\left(y-v_{0} t\right)+\frac{m}{4 \pi^{2}} \int_{-\infty}^{+\infty} \mathrm{d} k_{x}^{\prime} \int_{-\infty}^{+\infty} \mathrm{d} k_{y}^{\prime} \\
& \int_{-\infty}^{k_{x}^{\prime}} \mathrm{d} k_{x} \frac{\sqrt{k_{x}^{\prime 2}+k_{y}^{\prime 2}}}{\sqrt{k_{x}^{2}+k_{y}^{2}}} \tilde{\mathcal{R}}\left(k_{x}, k_{x}^{\prime}\right) \mathrm{e}^{-i\left[\frac{v_{0}}{2 A}\left(k_{x}-k_{x}^{\prime}\right)+k_{y}^{\prime} v_{0} t\right]} \\
& \times \mathrm{e}^{i\left[k_{x}^{\prime} x+k_{y}^{\prime} y\right]}
\end{aligned}
$$

where the explicit form of the $\omega$-dependent term of the resolvent operator $\mathcal{R}$ has been used (cf. Sect. 7). Again the first term of Eq. (116) represents the imposed moving point mass, while the second term is now proportional to $\mathrm{e}^{i k_{y}^{\prime}\left(y-v_{0} t\right)}$, and represents thus a travelling polarization cloud which is stationary in the reference frame moving along with the point mass.

\subsection{Response to white noise}

Toomre (1990) has argued that much of the recurrent spiral activity seen by him in his numerical sliding grid simulations of the dynamical evolution of a star disk or by Sellwood \& Carlberg (1984) in their numerical simulations is due to swing amplified random fluctuations induced by particle noise. In order to illustrate this effect I have subjected also the shearing sheet to such potential 
perturbations. I consider for this purpose the Fourier transform of the Volterra Eq. (68) with respect to time,

$\Phi_{\boldsymbol{k}^{\prime}, t}(t)=\int_{-\infty}^{k_{x}^{\prime}} \mathrm{d} k_{x} \tilde{\mathcal{K}}\left(k_{x}, k_{x}^{\prime}\right) \Phi_{k_{x}, k_{y}^{\prime}, t+\frac{k_{x}-k_{x}^{\prime}}{2 A k_{y}^{\prime}}}+r_{\boldsymbol{k}^{\prime}, t}$,

where use has been made of the explicit form of the $\omega$ dependent term of the kernel function and the convolution theorem of the Fourier transform of products of functions. This can be rewritten with the help of Eq. (90) in terms of the surface density

$\Sigma_{\boldsymbol{k}^{\prime}, t}=\sqrt{k_{x}^{\prime 2}+k_{y}^{\prime 2}} \int_{-\infty}^{k_{x}^{\prime}} \mathrm{d} k_{x} \frac{\tilde{\mathcal{K}}\left(k_{x}, k_{x}^{\prime}\right)}{\sqrt{k_{x}^{2}+k_{y}^{\prime 2}}} \Sigma_{k_{x}, k_{y}^{\prime}, t+\frac{k_{x}-k_{x}^{\prime}}{2 A k_{y}^{\prime}}}$

$+\Sigma_{\boldsymbol{k}^{\prime}, t}^{\mathrm{ext}}$

where $\Sigma_{\boldsymbol{k}^{\prime}, \mathrm{t}}^{\text {ext }}$ denotes the surface density associated with the randomly fluctuating potential perturbations.

In the case of white noise the autocorrelation function of the fluctuating surface density of the sheet is given by

$$
\begin{aligned}
& \left\langle\Sigma(\boldsymbol{x}, t) \Sigma^{*}\left(\boldsymbol{x}^{\prime}, t\right)\right\rangle=\int \mathrm{d}^{2} k \int \mathrm{d}^{2} k^{\prime}\left\langle\Sigma_{\boldsymbol{k}}(t), \Sigma_{\boldsymbol{k}^{\prime}}^{*}(t)\right\rangle \\
& \times \exp i\left[(\boldsymbol{k}, \boldsymbol{x})-\left(\boldsymbol{k}^{\prime}, \boldsymbol{x}^{\prime}\right)\right],
\end{aligned}
$$

where

$\left\langle\Sigma_{\boldsymbol{k}}(t), \Sigma_{\boldsymbol{k}^{\prime}}^{*}(t)\right\rangle=\left|\Sigma_{\boldsymbol{k}}(t)\right|^{2} \delta\left(\left(\boldsymbol{k}-\boldsymbol{k}^{\prime}\right) / k_{\mathrm{crit}}\right)$,

so that

$$
\begin{aligned}
& \left\langle\Sigma(\boldsymbol{x}, t) \Sigma^{*}\left(\boldsymbol{x}^{\prime}, t\right)\right\rangle \\
& =k_{\text {crit }}^{2} \int \mathrm{d}^{2} k\left|\Sigma_{\boldsymbol{k}}(t)\right|^{2} \exp -i\left(\boldsymbol{k}, \boldsymbol{x}-\boldsymbol{x}^{\prime}\right) \\
& =(2 \pi)^{2} k_{\text {crit }}^{2}\left|\Sigma_{\boldsymbol{k}}(t)\right|^{2} \delta\left(x-x^{\prime}\right) \delta\left(y-y^{\prime}\right),
\end{aligned}
$$

because the amplitudes $\Sigma_{\boldsymbol{k}}$ are uniformly distributed over wave number space. If the amplitude of the surface density fluctuations is expressed as fraction of the background density of the unperturbed sheet, $\varepsilon \Sigma_{0}$, one obtains

$$
\begin{aligned}
& \left\langle\Sigma(\boldsymbol{x}, t) \Sigma^{*}\left(\boldsymbol{x}^{\prime}, t\right)\right\rangle=\varepsilon^{2} \Sigma_{0}^{2} \\
& \approx k_{\text {crit }}^{2}\left|\Sigma_{\boldsymbol{k}}(t)\right|^{2} \frac{2 \pi}{\Delta x} \frac{2 \pi}{\Delta y}=k_{\text {crit }}^{2}\left|\Sigma_{\boldsymbol{k}}(t)\right|^{2} \Delta k_{x} \Delta k_{y},
\end{aligned}
$$

where the delta functions of Eq. (121) have been approximated according to the finite resolution of the numerical solution of the Volterra Eq. (118). The inhomogeneity of the integral equation is then given by

$\Sigma_{\boldsymbol{k}}^{\mathrm{ext}}=\left[\frac{\varepsilon^{2} \Sigma_{0}^{2}}{k_{\mathrm{crit}}^{2} \Delta k_{x} \Delta k_{y}}\right]^{\frac{1}{2}} \cdot \cos (\phi)$,

defined here as a real quantity, and where $\phi$ denotes a random angle distributed uniformly over the range $[0,2 \pi]$. Finally it is advantageous to write the integral Eq. (118) and expression (123) in dimensionless form by dividing them by the background density, which in wave number space is given by $\Sigma_{0} \delta(\boldsymbol{k}) \approx \Sigma_{0} /\left(\Delta k_{x} \Delta k_{y}\right)$. Figure 6 shows a typical solution of Eq. (118). The parameters of the disk
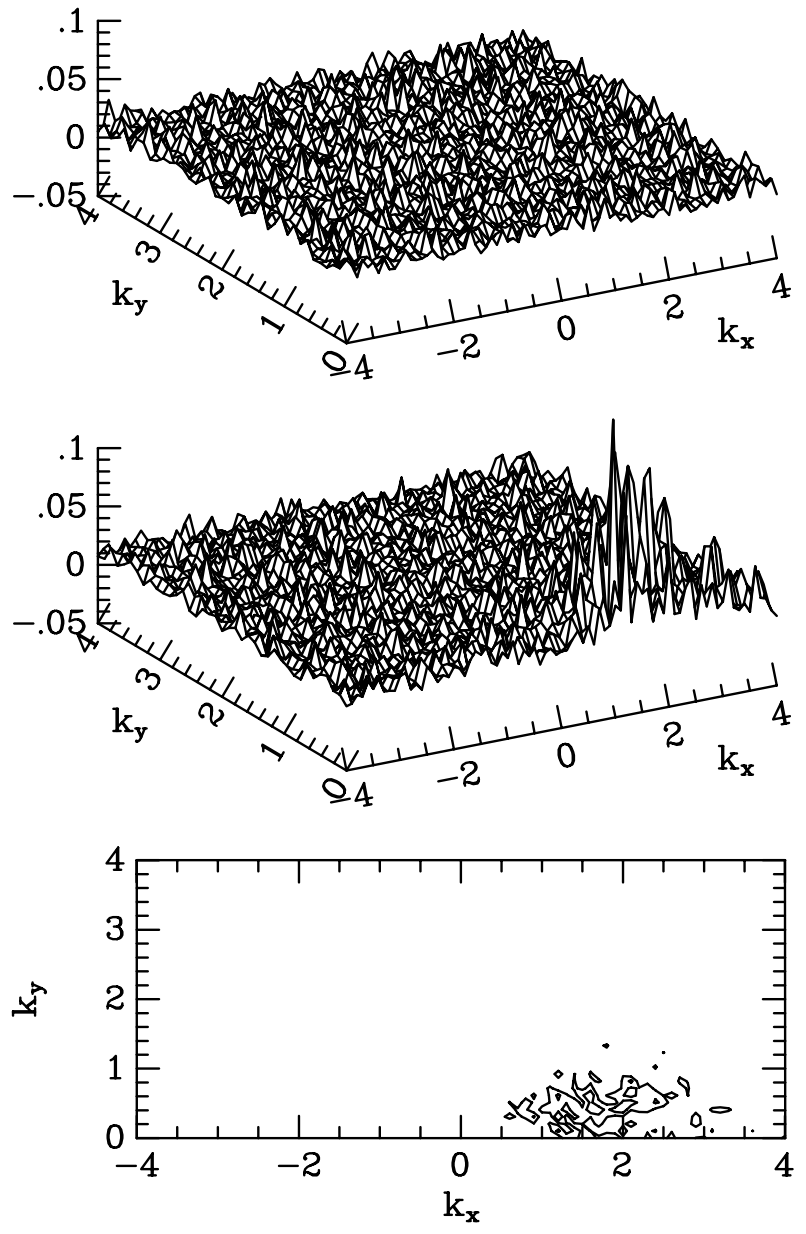

Fig. 6. Response of the swing amplifier to white noise input. The amplitude of the surface density, $\sqrt{\left|\Sigma_{\boldsymbol{k}}\right|^{2}}$, is plotted versus wave numbers $k_{x}$ and $k_{y}$. The upper panel shows a snapshot of the ever present, externally imposed white noise fluctuations. The middle panel shows the quasi-stationary equilibrium reached after the perturbations of the shearing sheet have fully developed. The lower panel shows a contour plot of the quasistationary equilibrium distribution. The parameters of the disk model are chosen as $A / \Omega_{0}=0.5$ and $Q=1.4$

model are adopted again as $A / \Omega_{0}=0.5$ and $Q=1.4$. The time steps at which solutions of Eq. (118) can be calculated are given by multiples of

$\Delta t=\frac{\Delta k_{x}}{2 A k_{y}^{\prime}}$

depending on the circumferential wave number $k_{y}^{\prime}$. Thus solutions are obtained at different time intervals. However, within less than an orbital period a quasi-stationary equilibrium is reached, which is shown in the second panel of Fig. 6. Each spike represents the amplitude $\sqrt{\left|\Sigma_{\boldsymbol{k}}\right|^{2}}$ of the superposition of the randomly incited swing-amplified density waves, all travelling from left to right at a velocity of $\dot{k}_{x, \text { eff }}=2 A k_{y}$. The contour plot in Fig. 6 shows clearly the peak of amplitudes around $\left(k_{x}, k_{y}\right) \approx(1.7,0.5)$ $k_{\text {crit }}$. This corresponds exactly to the amplification factors shown in Fig. 1.

It was already noted in the numerical simulations by Sellwood \& Carlberg (1984) that the constant spiral 
activity leads to dynamical heating of the disk, because the stars are randomly accelerated by the shearing density waves. This implies a rapid increase of the velocity dispersion of the stars and thus of the stability parameter $Q$ (cf. Eq. (95)), which will eventually suppress any spiral activity, if the disk is not dynamically cooled. Disk heating by swing amplified density waves in the shearing sheet will be discussed in detail in a further paper of this series (Fuchs 2001, in preparation).

\section{Summary and conclusion}

The objective of this paper is to present a new description of the dynamics of a shearing sheet made of stars. It is shown again that disturbances of the disk evolve always into swing amplified density waves, i.e. spiral-arm like, shearing density waves, which grow and decay while their wave crests swing by from leading to trailing orientation. Specific examples are discussed how such swing amplification events are incited. Only in response to imposed massive perturbers the shearing sheet develops stationary perturbations.

Acknowledgements. I thank A. Toomre and R. Wielen for their advice over the years. Helpful discussions with E. Athanassoula, N.W. Evans, J. Sellwood, and M. Tagger are gratefully acknowledged.

\section{Appendix}

Comparison of Eqs. (89) and (87) shows that the amplitude function in Eq. (89) can be calculated directly from the solution of the Volterra integral Eq. (68) with the inhomogeneity (86), if $k_{x}^{\prime}$ takes the role of $k_{x}^{\text {in }}+2 A k_{y}^{\text {in }} t$, $\left(k_{x}^{\prime} \geq k_{x}^{\prime \text { in }}\right)$, and $\mathcal{K}$ and $\mathcal{L}$ are replaced by $\tilde{\mathcal{K}}$ and $\tilde{\mathcal{L}},(\omega=0)$,

$\Phi_{k_{x}^{\prime}, k_{y}^{\prime}}=\int_{k_{x}^{\mathrm{in}}}^{k_{x}^{\prime}} \mathrm{d} k_{x} \tilde{\mathcal{K}}\left(k_{x}, k_{x}^{\prime}\right) \Phi_{k_{x}, k_{y}^{\prime}}+\tilde{\mathcal{L}}\left(k_{x}^{\mathrm{in}}, k_{x}^{\prime}\right)$.

Equation (125) is discretized as $\left(k_{x}^{\prime}=k_{j}^{\prime}\right)$

$$
\begin{aligned}
& \int_{k_{i}}^{k_{i}+1} \mathrm{~d} k_{x} \tilde{\mathcal{K}}\left(k_{x}, k_{x}^{\prime}\right) \Phi\left(k_{x}, k_{y}^{\prime}\right)= \\
& \frac{1}{2}\left[\tilde{\mathcal{K}}\left(k_{i}+1, k_{j}^{\prime}\right) \Phi\left(k_{i}+1, k_{y}^{\prime}\right)\right. \\
& \left.\quad+\tilde{\mathcal{K}}\left(k_{i}, k_{j}^{\prime}\right) \Phi\left(k_{i}, k_{y}^{\prime}\right)\right] \Delta k
\end{aligned}
$$

leading to

$$
\begin{aligned}
& \Phi\left(k_{j}^{\prime}, k_{y}^{\prime}\right)=\sum_{k_{i}=-\infty}^{k_{j}^{\prime}-1} \frac{\Delta k}{2}\left[\tilde{\mathcal{K}}\left(k_{i}+1, k_{j}^{\prime}\right) \Phi\left(k_{i}+1, k_{y}^{\prime}\right)\right. \\
& \left.+\tilde{\mathcal{K}}\left(k_{i}, k_{j}^{\prime}\right) \Phi\left(k_{i}, k_{y}^{\prime}\right)\right]+\tilde{\mathcal{L}}\left(k_{x}^{\mathrm{in}}, k_{j}^{\prime}\right) .
\end{aligned}
$$

According to the definition of the kernel $\tilde{\mathcal{K}}\left(k_{j}^{\prime}, k_{j}^{\prime}\right)=0$, so that the set of algebraic Eqs. (127) can be resolved recursively:

$$
k_{j}^{\prime}<k_{x}^{\text {in }}: \Phi\left(k_{j}^{\prime}, k_{y}^{\prime}\right)=0,
$$

$$
\begin{aligned}
& k_{j}^{\prime}=k_{x}^{\text {in }}: \Phi\left(k_{j}^{\prime}, k_{y}^{\prime}\right)=\tilde{\mathcal{L}}\left(k_{x}^{\text {in }}, k_{x}^{\text {in }}\right) \\
& k_{j}^{\prime}=k_{x}^{\text {in }}+1: \Phi\left(k_{j}^{\prime}, k_{y}^{\prime}\right)=\left[\tilde{\mathcal{K}}\left(k_{x}^{\text {in }}-1, k_{x}^{\text {in }}+1\right) \Phi\left(k_{x}^{\text {in }}-1, k_{y}^{\prime}\right)\right. \\
& +\tilde{\mathcal{K}}\left(k_{x}^{\text {in }}, k_{x}^{\text {in }}+1\right) \Phi\left(k_{x}^{\text {in }}, k_{y}^{\prime}\right)+\tilde{\mathcal{K}}\left(k_{x}^{\text {in }}, k_{x}^{\text {in }}+1\right) \Phi\left(k_{x}^{\text {in }}, k_{y}^{\prime}\right) \\
& \left.+\tilde{\mathcal{K}}\left(k_{x}^{\text {in }}+1, k^{\text {in }}+1\right) \Phi\left(k_{x}^{\text {in }}+1, k_{y}^{\prime}\right)\right] \frac{\Delta k}{2} \\
& +\tilde{\mathcal{L}}\left(k_{x}^{\text {in }}, k_{x}^{\text {in }}+1\right) \\
& =\tilde{\mathcal{L}}\left(k_{x}^{\text {in }}, k_{x}^{\text {in }}+1\right)+\tilde{\mathcal{K}}\left(k_{x}^{\text {in }}, k^{\text {in }}+1\right) \Phi\left(k_{x}^{\text {in }}, k_{y}^{\prime}\right) \Delta k \\
& k_{j}^{\prime}>k_{x}^{\text {in }}: \Phi\left(k_{j}^{\prime}, k_{y}^{\prime}\right)=\tilde{\mathcal{L}}\left(k_{x}^{\text {in }}, k_{j}^{\prime}\right) \\
& \quad k_{j}^{\prime}-1 \\
& +\sum_{k_{i}=k_{x}^{\text {in }}} \tilde{\mathcal{K}}\left(k_{i}, k_{j}^{\prime}\right) \Phi\left(k_{i}, k_{y}^{\prime}\right) \Delta k .
\end{aligned}
$$

In the numerical realization of the scheme I have adopted a stepwidth of $\Delta k=0.1 k_{\text {crit }}$.

\section{References}

Abramowitz, M., \& Stegun, I. A. 1968, Handbook of Mathematical Fucntions (Dover, New York)

Athanassoula, E. 1984, Phys. Rep., 114, 319

Binney, J., \& Tremaine, S. 1987, Galactic Dynamics (Princeton Univ. Press, Princeton)

Bertin, G., Lin, C. C., Lowe, A., et al. 1989a, ApJ, 338, 78

Bertin, G., Lin, C. C., Lowe, A., et al. 1989b, ApJ, 338, 104

Donner, K. J., \& Thomasson, M. 1994, A\&A, 290, 785

Fuchs, B. 1991, in Dynamics of Disc Galaxies, ed. B. Sundelius (CTH Göteborg), 359

Goldreich, P., \& Lynden-Bell, D. 1965, MNRAS, 130, 125

Goldreich, P., \& Tremaine, S. 1978, ApJ, 222, 850

Gradshteyn, I. S., \& Ryzhik, I. M. 1965, Table of Integrals, Series, and Products, 4th Ed. (Academic Press, New York)

Hunter, C. 1992, in Astrophysical Disks, ed. S. F. Dermott, J. H. Hunter, \& R. E. Wilson (New York Academy of Sciences)

Julian, W. H., \& Toomre, A. 1966, ApJ, 146, 810

Kalnajs, A. J. 1971, ApJ, 166, 275

Kalnajs, A. J. 1977, ApJ, 212, 637

Kalnajs, A. J. 1978, in Structure and Properties of Nearby Galaxies, ed. E. M. Berkhuisen, \& R. Wielebinski (Reidel, Dordrecht), 113

Lin, C. C. 1970, in Galactic Astronomy, vol. II, ed. H. Y. Chiu, \& A. Muriel (Gordon and Breach, New York), 1

Lin, C. C., Yuan, C., \& Shu, F. H. 1969, ApJ, 155, 721

Lindblad, B. 1959, in Handbuch der Physik, vol. LIII, ed. S. Flügge (Springer, Berlin), 21

Lowe, S. A., Roberts, W. W., Yang, J., et al. 1994, ApJ, 427, 184

Morse, P. M., \& Feshbach, H. 1953, Methods of Theoretical Physics (McGraw Hill, New York)

Pichon, C., \& Cannon, R. C. 1997, MNRAS, 291, 616

Sellwood, J. A., \& Carlberg, R. G. 1984, ApJ, 282, 61

Sellwood, J. A., \& Evans, N. W. 2000 [astro-ph/0006198]

Toomre, A. 1981, in The Structure and Evolution of Normal Galaxies, ed. S. M. Fall, \& D. Lynden-Bell (Cambridge Univ. Press, Cambridge), 111

Toomre, A. 1990, in Dynamics and Interactions of Galaxies, ed. R. Wielen (Springer, Berlin), 292

Toomre, A., \& Kalnajs, A. J. 1980, private memorandum

Toomre, A., \& Kalnajs, A. J. 1991, in Dynamics of Disc Galaxies, ed. B. Sundelius (CTH Göteborg), 341 